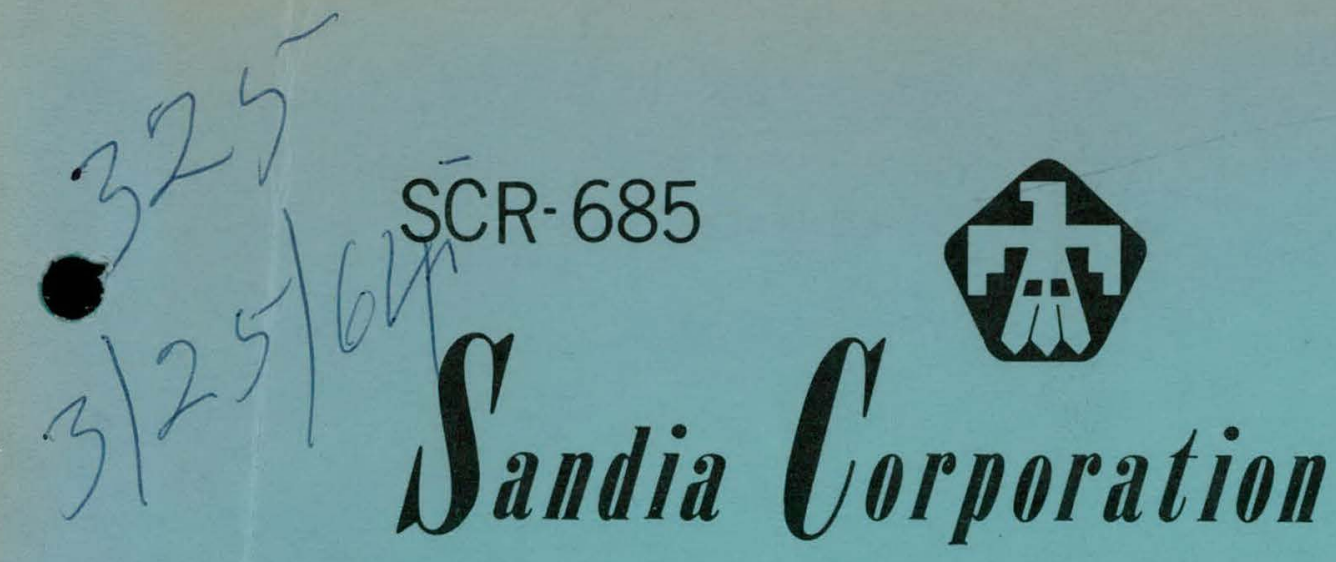

•....... MONOGRAPH

\title{
A METHOD FOR PERFORMING A \\ HUMAN-FACTORS RELIABILITY ANALYSIS
}

by

A D Swain

August 1963 


\section{DISCLAIMER}

This report was prepared as an account of work sponsored by an agency of the United States Government. Neither the United States Government nor any agency Thereof, nor any of their employees, makes any warranty, express or implied, or assumes any legal liability or responsibility for the accuracy, completeness, or usefulness of any information, apparatus, product, or process disclosed, or represents that its use would not infringe privately owned rights. Reference herein to any specific commercial product, process, or service by trade name, trademark, manufacturer, or otherwise does not necessarily constitute or imply its endorsement, recommendation, or favoring by the United States Government or any agency thereof. The views and opinions of authors expressed herein do not necessarily state or reflect those of the United States Government or any agency thereof. 


\section{DISCLAIMER}

Portions of this document may be illegible in electronic image products. Images are produced from the best available original document. 


\author{
Published by \\ Sandia Corporation, \\ a prime contractor to the \\ United States Atomic Energy Commission
}

\title{
LEGAL NOTICE
}

This report was prepared as an account of Government sponsored work. Neither the United States, nor the Commission, nor any person acting on behal of the Commission:

A. Makes any warranty or representation, expressed or implied, with respect to the accuracy, sompleteness, or usefulness of the information contained in this report, or that the use of any information, apparatus, method, or process disclosed in this report may not infringe privately owned rights; or

B. Assumes any liabilities with respect to the use of, or for damages resulting from the use of any information, apparatus, method, or process disclosed sulting from the use of any information, apparatus, method, or process disclosed
in this report. in this report.

As used in the above, "person acting on behalf of the Commission" includes any employee or contractor of the Commission, or employee of such contractor. to the extent that such employee or contractor of the commission or employee of such contractor prepares, disseminates, or provides access to, any information pursuant to his employment or contract with the Commission, or his employment with such contractor.

Printed in USA. Price $\$ 1.50$. Available from the Office of Technical Services, Department of Commerce, Washington 25, D. C. 
SCR- 685

ENGINEERING AND EQUIPMENT

TID -4500 (26th Ed.)

SANDIA CORPORATION MONOGRAPH

A METHOD FOR PERFORMING A HUMAN-FACTORS RELIABILITY ANALYSIS

by

A. D. Swain

August 1963 
A method for performing a human-factors reliability analysis of a man-machine system is described. The outcome of this lype of analysis is a quantitative assessment of the estimated degradation of a man-machine systcm resulting from human crrors. The methor represents an extension of a quantitative approach to reduction of human error in industrial production described by $\mathrm{L}$. W. Rook, Jr., in Reduction of Human Error in Industrial Production, SCTM 9.3-62(14). 


\section{FOREWORD}

There has been relatively little work reported on deriving quantitative estimates of the degradation to a man-machine system resulting from human errors. One approach, used in the Reliability Department of Sandia Corporation, is presented here as an example of how such estimating can be done. It is hoped that this approach will be of use to human-factors specialists and others interested in the effects of human factors on systems.

The approach presented herein uses probabilistic models and probability techniques. Both the models and techniques are elementary for those with experience or training in the theory of probability. However, since many human-factors specialists have had little training in these areas, the discussions and examples related to the application of probability theory are pitched at an elementary level. If the reader will take the time and effort to go through the mathenatical models, he will have acquixed at least the initial skills in estimating system effects of human crrors.

Acknowledgment is due F. W. Müller, Supervisor, Reliability Training and Development Section, Sandia Corporation, for his original contributions to the probabilistic approach, for his development of certain mnemonic aids for constructing reliability equations, and for his patience in checking the equations in this report. Mr. Muller and Mr. J. M. Wiesen, Manager, Reliability Department, Sandia Corporation, worked out a method for deriving the individual equations for allocating the appropriate portion of system-failure rate among the human errors in the system.

The approach also uses a data bank of figures for estimating the probability that any given behavioral dimension of a human action will not lead to incorrect performance of that action. This unique data bank was developed by Dr. J. W. Altman and his colleagues at the American Institute for Research.

Fina11y, acknowledgment is due Dr. L. W. Rook, Jr., Advanced System Studies, Sandia Corporation, who has described a quantitative approach to reduction of human error in industrial production. This report extends Rook's quantitative approach to military operational tasks and jobs.

The present report was prepared in part as the author's contribution to Task Group 2 - Quantification of Human Parformance, in the M-5.7 Military Subcommittee on Human Factors in Electronics, of the Electronics Industries Association. Task Group 2 is made up of the following human-factors specialists: Dr. Alvyn Freed, Aerojet-General Corporation, Dr. Melvin Freitag, Ryan Aeronautical Company, Dr. Charles A. Fenwick, Coliins Radio Company, Mr. Robert Kinch, Western Electric Company, Dr. Edmund T. Klemmer, Bell Telephone Laboratories, Mr. Harald R. Leuba, ARINC Research Corporation, Captain Melvin S. Majesty, Hq. Ballistic Systems Division, USAF, Dr. David Meister, General Dynamics/Astronautics, and Dr. Alan D. Swain, Sandia Corporation (Chairman). Persons interested in quantifying predictions of human performance may contact any of the above men for further information on the work of Task Group 2, EIA, M-5.7. 
TABLE OF CONTENTS

CHAPTER I

AN OVERVIEW OF $\cdot$ THE METHOD . . . . . . . . . . . . . . . . . . . 7

Introduction... . . . .... . . . . . . . . . . . . . . . . . . . . . . 7

Technique for Human-Exror Rate Prediction . . . . . . . . . . . . . . . 8

Define System Failure . . . . . . . . . . . . . . . . . . . . . . 9

List the Human Operations. . . . . . . . . . . . . . . . . . . . 9

Predict Individual Error Rates . . . . . . . . . . . . . . . . . . . . . . . . . . 9

Determine the Effects of Human Errors on the System . . . . . . . 13

Recommend Changes to System and Calculate New System

Failure Rate . . . . . . . . . . . . . . . . . . . . . 15

CHAPTER II $\triangle$ PROCEDURE $\triangle$ ND SOME ASSUMPTIONS FOR ESTIMATING ERROR RATES . . 17

Introduction . . . . . . . . . . . . . . . . . . . . . . . . . . . . 17

Use of AIR Data. . . . . . . . . . . . . . . . . . . . . . . . . . . 17

An Assumption for Monitored Behavior . . . . . . . . . . . . . . . . . 19

An Assumption for Sclf-Correction of Errors . . . . . . . . . . . . . . 20

An Assumption for B̈ehavior Under High-Stress Conditions . . . . . . . . 21

CHAPTER III A PROCEDURE TO ESTIMATE THE EFFECTS OF HUMAN ERRORS ON A

MAN-MACHINE SYSTEM . . . . . . . . . . . . . . . . . . 25

Introduction . . . . . . . . . . . . . . . . . . . . . . . . . . . . . 25

A Hypothetical Operational Situation . . . . . . . . . . . . . . . . . 25

Assumed Operational Sequence . . . . . . . . . . . . . . . . 25

Major Assumptions . . . . . . . . . . . . . . . . . . . . . . . . 26

Situation Diagram . . . . . . . . . . . . . . . . . . . . . 27

Probability Estimates . . . . . . . . . . . . . . . . . . . . 27

Calculation of Estimated System Degradation Resulting from

Human Error . . . . . . . . . . . . . . . . . . . . . . . . . . . . . 29

Direct Versus Indirect Approach . . . . . . . . . . . . . . . . . 29

The Indirect Approach . . . . . . . . . . . . . . . . . . . . . . 32

The Disecl Appivacli . . . . . . . . . . . . . . . . . . . . . . . J0

An Approximation of the Exact Failure Equation . . . . . . . . . . 38

Recommend Changes to System and Calculate New System Failure Rate . . 41

Calculation of $Q_{i}$ Values . . . . . . . . . . . . . . . . . . . : 41

Diocuooion of $Q_{i}$ Valucc . . . . . . . . . . . . . . . . . . . . 18

CHAPTER IV SUMMARY AND CONCLUSIONS . . . . . . . . . . . . . . . . . . . . . 50

REFERENCES . . . . . . . . . . . . . . . . . . . . . . . . 51

\section{LIST OF ILLUSTRATIONS}

\section{Figure}

1. Situation diagram of hypothetical military sequence . . . . . . . . 27

2. Probability tree illustrating branching technique . . . . . . . . . 32 
I. Time and Success Probability Estimates for Input Component "Labeling" . . . . . . . . . . . . . . . . . . . . . . . 11

II. Time and Success Probability Estimates for Output Component "Speaking" . . . . . . . . . . . . . . . . . . . . . . 19

III. Probability Estimates for Hypothetical Military Sequence . . . . . . 28

IV. Mnemonic Aids for Probability Equations . . . . . . . . . . . . . . . 30

V. The Failure Paths Due to Human Error . . . . . . . . . . . . . . . 45

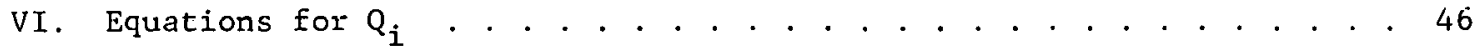

VII. Hypothetical $Q_{i}$ Values and Rank Order from High to Lo'N . . . . . . . 47

VIIT. Ranks of High and Low Hypothetical $Q_{i}$ Values . . . . . . . . . . . . 48 


\section{CHAPTER I}

\section{AN OVERVIEW OF THE METHOD}

Introduction

At the 9th Military Operations Research Symposium (MORS) in early 1962, L. W. Rook, Jr., Sandia Corporation, described a method for evaluating the human error contribution to system degradation (Reference 1). This method is based partly on research on human reliability by J. W. Altman and his associates (see References 2, 3, 4, and 5) at the American Institute for Research (AIR), on research done by Rook at Sandia Corporation, and upon probability theory and other methods used by reliability engineers. This method, now called THERP (Technique for HumanError Rate Prediction), is more fully describcd in Reference 6 .

Referencoc 1 and 6 , however, present the methud in the concext of predicting industrial errors and controlling degradation resulting from human errors in industrial processes. The method described in these references can also be applied to military duties in a field operational setting. However, the mathematical treatment of THERP is brief, and fuller treatment of both the mathematics and the application of THERP to field operations seems desirable. These are the primary purposes of this report.

In order to avoid security classification problems, only hypothetical job situations are discussed in illustrating the technical approach. For the reader with a security clearance, there are available two additional classified references showing the application of THERP by Sandia personnel to a military classified problem (References 7 and 8 ) .

\footnotetext{
* Reference 7 should be adequate for readers interested in human factors only. Reference 8 includes estimates of equipment reliability and total system reliability but does not contain the detailed human-factors discussion in Reference 7.
} 
Technique for Human-Error Rate Prediction

A technique used in the Reliability Department of Sandia Corporation, THERP predicts human-error rates in a man-machine system* and evaluates the degradation to the system or any part of it likely to be caused by human errors* in association with equipment reliability, operational procedures, and other system characteristics which influence human behavior. Finally, THERP is an iterative procedure, consisting of five steps which are repeated, not always in the same order, until the system degradation resulting from human error is at an acceptable level. The five steps are listed below and described more fully on the following pages.

1. Define the system or subsystem failure which is to be evaluated.

2. Identify and list all the human operations performed and their relationships to system tasks and functions.

3. Predict error rates for each human operation or group of operations pertinent to the evaluation.

4. Determine the effect of human errors on the systen.

5. Recommend changes as necessary to reduce the system or subsystem failure rate as a consequence of the estimated effects of the recommended changes.

Although most human-factors studies of man-machine systems do not attempt to predict error rates, it is apparent that there is nothing basically new in these five general steps. The steps are typical of the usual system reliability study if one substitutes "hardware" for "numans." In fact, as long as ten years ago (Reference 9), this general procedure was applied by Sandia Corporation in estimating the degradation to a nuclear weapon system resulting from the effects of human errors. However, since that time, the procedure has been refined, especially in steps 2, 3, and 5. Moreover, some new developments, for example the AlK method of analyzing the reliability of human-behavior components (Reference 3) and the Sandia Corporation human-error classification scheme (Reference 6), have made this whole quantitative approach to human factors more reliable and thus of greater value to system designers.

*A man-machine system is an arrangement of men and equipment for achieving one or more goals by correctly performing functions and tasks related to each goal. The system may be broken down into subsystems or part-systems, each of which has its functions, tasks, and goals. In this report, it is often convenient to use the word "system" either to denote a complete man-machine system or any part of it.

HHunan errors occur (1) when a man fails to perform a task or a part of a task (e.g., a step), (2) when he performs the task or step incorrrectly (3) when he introduces some task or step which should not have been performed, (4) when he performs some task or step out of sequence, or (5) when he fails to perform the task or step within the alloted time period. The effect of hunan errors in reducing the probability of the system achieving its goals is ascertained by the use of the probabilistic model described in this report. From a "system" point of view, a human behavior is an error only when it has the potential effect of reducing system or part-system reliability or otherwise reducing the likelihood that some system or part system success criterion will be met. 


\section{Define System Failure}

The first step in the application of THERP is to define the system (or partsystem) failure which is to be evaluated. This failure may vary from (1) such distal criteria in the life of a weapon as the probability of the weapon failing to explode within a certain area and altitude at a certain time to (2) more proximal criteria such as the failure of a component in the weapon to meet engineering specifications. As stated above, the human-factors evaluation is restricted to the influences of the human element on system failure and therefore identifies only part of the total unreliability of the system. This restriction does not remove the interactions of machine and human influences from the evaluation, but it does eliminate consideration of the influences of machine variables per se.

\section{List the Human Operations}

The next step is to identify and list all the human operations performed and their relationships to system tasks and functions. System and task analysis (Reference 10), a method familiar to human-factors specialists, is the basic method used in this step. Variations of this procedure are sometimes used (see Reference 11).

The system and task analysis must uncover all those possible human actions and procedures which can enter into the evaluation. They include whatever human procedures are: required in the event of equipment defects or breakdowns or to compensate for other abnormal conditions. They also include human operations which are not a part of the prescribed operating procedure but which might be substituted for the required human operations.

At this point, the analyst ordinarily makes some restriction in the human operations to be considered further in the reliability analysis. That is, he drops from consideration those human operations for which it is apparent that no significant degradation to system or part-system failure rate would result as a function of their incorrect performance. Errors in human operations judged to be not relevant to this failure rate are not errors in the context of the system being evaluated. It is best to be very conservative in dropping from the analysis those human operations judged to be irrelevant to system success. If an erroneous judgment is made at this point, the entire reliability analysis can be highly misleading and the resultant recommendations (or lack of recommendations) could have a highly detrimental effect on the design and use of the system.

\section{Predict Individual Error Rates}

Next, error rates are estimated for each human operation relevant to system failure rate. Judgments are made or data are obtained on the nature of the interactions of correct and incorrect human operations in the context of the systeill. Finally, as discussed in the next section, the error rates are appropriately combined according to conventional probability theory. The estimates of error rates draw on actual human-error-rate data whenever available and appropriate. Two major sources of human-error-rate data are used at Sandia: (1) experimental or other empirical data and (2) the AIR Data Store (Reference 2). 
The question of appropriateness of data is an important one. Usually, experimental data from behavioral laboratories in university psychology departments cannot be generalized directly to an operational situation. First of all, university experiments are often set up to generate high error rates in order to minimize the number of subjects necessary to test the statistical significance of measurable differences between experimental conditions or treatments. In the usual operational situation, on the other hand, error rates are ordinarily much lower--often at least two orders of magnitude lower. A second reason for the disparity in error rates is that the operational situation is ordinarily multitask, e.g., the task in which an investigator is interested is but one of many tasks which are competing for the attention of an operator. The nature of the competition and the frequency per unit time nf the task of interest markedly affect the error rate and could make it either larger or smaller than the error race frull d culsespundiug labsratory cupcriment. A third reason is that stress conditions in the laboratory situation often bear little resemblance to high-stress conditions found in certain operatiunal situations. Generally, under high-stress conditions (as defined in Chapter II) behavior accuracy and relevancy can be expected to deteriorate grossly.

A11. of these factors point to the need for the utmust cauliull ill geileralizing from laboratory data to the operational situation. However, error rates drawn from operational suitability tests or other test situations which more closely approximate operational conditions can be used with more confidence In the absence of a central data bank (or data store) of human-error rates and associated environinental and other conditions (as recommended in Reference 12), the human-factors specialist who wishes to predict error rates often must develop h1s own empirical data At the present time, considerable judgment is required, and, in this sense, quantification of predictions of human behavior is an art as well as a science. Even so, experience at Sandia has indicated that predictions of human-error rates made by the two Sandia human-factors specialists disagree by no more than abuul 30 peicent.

A good start on a data bank of human-error rates has been made by AIR (Reference 2). This is the "data store" to the "Index of electronic equipment operability." The AIR Data Store uses a specific step or action in a given task as the basic unit of evaluation. Each spec1f1c scep ur deliun is broken down into three aspects: (1) inputs or stimuli to the human senses, (2) mediating processes (making decisions, inferences, interpretations, or judgments, recalling information, anticipating future events, etc.), and (3) outputs or responses (e.g., motor responses of the human). In deriving this data store, major types of equipment components, behavior components, or equipment-behavior components likely to affect each aspect were identified. For example, under input (stimulus) aspects are the following components: circular scales, counters, labeling, lights, linear scales, nonspeech, scopes, semicircular scales, and speech. Under the input component "labeling" (see Table I) are three parameters: span, legibility, and size of printing. Each of these parameters is broken down into dimensions; i.e.; physical descriptions of the component concerned. For example, the parameter "span" is broken down into four "digit" dimensions and three "word" dimensions. Opposite each of these dimensions appear a "time added" figure in seconds and a "success probability" figure which denotes the probability that the dimension in question 
will not contribute to the error rate of the step or action containing that dimension. (This report uses the more exact term "success probability figure" in place of the AIR term "reliability" which is subject to misinterpretation by reliability specialists.) This dimensional approach recognizes that human reliability is a function of the compatibility of equipment design with the capabilities and limitations of the operator, even when he is well-trained.

TABLE I

Time and Success Probability Estimates for Input Component "Labeling" (Taken from Reference 2)

BASE TIME - 0.20 seconds

Time added Success probability (seconds)

0

0.11

0.29

0.71

0

0.20

1.65

0

0.25

0

0.20
0.9997

0.9998

0.9992

0.9991

0.9999

0.9995

0.9985

0.9999

0.9997

0.9994
0.9994
Labeling

(Includes any labeling serving as the step input)

1. Span

a. Digits

(1) 2

(2) 3

(3) $4-5$

(4) $6-7$

b. Words

(1) 1 or 2

(2) $3-5$

(3) 6-11

2. Legibility

a. Clear and suncise

b. Potentially ambiguous

3. Size of printing (height)

a. $1 / 5^{\prime \prime}$ or more

b. $1 / 8^{\prime \prime}$

The complement of (i.e., one minus) the product of all of the relevant success probability figures for the inputs, mediating processes, and outputs for an action is the estimated error rate for that action. The sum of all of the relevant time figures for the inputs, mediating processes, and outputs for an action is the estimated time required to complete that action.

The time and success probability figures in the AIR Data Store are based on time measures and error rates from many experimental studies with a correction factor (approximately .008 x experimental error rate) applied to account for the differences between the laboratory and operational (field) situations. Both time and success probability figures assume equipment at least moderately well engineered from the human-factors standpoint and average motivation and adequate training for the operator. If the human engineering of the equipment is poor. (including, for example, violations of strong populational stereotypes), then the figures in the data store would grossly underestimate both time and error-rate figures. Poor motivation or inadequate training could also result in gross inaccuracies in the 
estimated time and error-rate figures. However, as a rule of thumb, Sandia humanfactors specialists have considered that poor human engineering can often result in error-rate increases of one or more orders of magnitude, but under usual operational conditions, they have concluded that poor motivation and training ordinarily degrade human accuracy by no more than a maximum of a factor of 5. (See the discussion on pages 8 and 9 of Reference 6. )

Chapter II illustrates the way in which Sandia Corporation human-factors specialists have used the AIR Data Store. The example in Chapter II is hypothetical, but the behaviors involved in the example and the interpretations of data made by the author are representative of those involved in the operational study described in Reference 7 .

Chapter II makes it apparent that considerable judgment is involved in accepting the figures from the AIR Data Store, especially in judging when behaviors are or are not independent* or in deciding whether the effects of stress preclude the use of the data store. Moreover, error rates derived from the data store are only as good as the basic success probability figures contained in the data store. Not nearly enough validation of predictions based on the AIR Data Store has been done. However, some comparisons. of actual error rates in certain factory assembly and inspection operations with predicted error rates using the AIR Data Store have been made at Sandia. It has been found that the predicted error rates differed by no more than a factor of 3 or 4 at the most. For many applications, this degree of accuracy is sufficient. At Sandia Corporation, for example, no more accuracy in prediction than a factor of five has been claimed in human-factors reliability studies.

It is important to reiterate that the estimates of error rates derived from the AIR Data Store are for the "average military operator" trained in the operation in question and with "average" motivation and under "normai" operating conditions. (The use of quotation marks is intended to connote an unknown degree of inaccuracy.) Ordinarily, the average error rates are not accompanied with estimates of standard deviations or other measures of dispersion about the average (mean). Thus, estimated error rates based on the AIR Data Store or on other sources of data can be grossly in error when applied lu a given individual. The error rates are estimates of the average error rate for an individual selected at random from the group of operators being studied. Therefore, in the Sandia Corporation humanfactors reliability analyses, the assumptions state the level of stress, type of environmental conditions, the level of motivation (but not usually including malicious deviations from a standard operating procedure), and the type and extent of training. (in general terms) that the operators are assumed to have had. Furthermore, the human-factors specialists feel an obligation to explain to the users of the reliability analyses the nature of the basic human-error-rate data which enter into the estimates. This is especially true in the case of estimates of humanerror rates under high-stres's conditions (as defined in Chapter II).

* THERP makes no assumptions as to the dependence or independence of behaviors. It is up to the user of THERP to make these assumptions and to use. probability equations consonant with his assumptions. 
The lack of precision that the above paragraph denotes is certainly not a satisfactory state of affairs. Perhaps this section of the report illustrates best why those of us who have to make estimates of human reliability hope that a large scale effort of the kind recommended in Reference 12 will soon get underway to begin the growth of the much needed computerized data bank of human-error-rate figures, including related standard deviations, characteristics of operators, and environmental and other factors affecting error rates.

\section{Determine the Effects of Human Errors on the System}

Having determined the error rates, it is necessary to determine the probability $\left(F_{i}\right)^{*}$ that each error, error class, or group of errors which have occurred will result in a failure of the system or that part of the system being evaluated. The probability of a failure condition resulting from a single incorrect performance of a human operation of Class $i$ is then the joint probability $F_{i} P_{i}$, where $P_{i}$ is the probability that an operation can and 1 s supposed to occur and that it will lead to an error of Class i. A group of incorrect human operations, or incorrect human operations plus equipment defects or other factors, may be necessary for the failure condition to occur; $P_{i}$ can also stand for the probability of these conditions.** It may be that a human operation or series of operations performed more than once and a single incorrect performance results in a failure condition with probability $F_{i}$. In this case we would be interested in the probability that $n_{i}$ operations will result in one or more failure conditions because of errors of class $i$. This probability can be evaluated as 1 minus the probability of no failure conditions from this source; it is given by $Q_{i}=1-\left(1-F_{i} P_{i}\right)^{n_{i}}$, where $Q_{i}$ is the probability of one $u i$ mure fallure conditions existing as a result of class $i$ errors occurring in $\mathrm{n}_{\mathrm{i}}$ operations. ${ }^{\dagger \dagger}$ The total system (or subsystem) failure rate resulting from human error can be expressed as $Q_{T}=1-\left[\prod_{k=1}^{n}\left(1-Q_{k}\right)\right]^{\dagger}$ where $Q_{T}$ is the probability that one or more failure conditions will result from errors in at least one of $n$ classes of errors, and the quantity in brackets represents $\left(1-Q_{1}\right)\left(1-Q_{2}\right) \ldots\left(1-Q_{n}\right)$.

\footnotetext{
${ }^{*} \mathrm{P}_{i}, \mathrm{~F}_{i}, \mathrm{Q}_{i}, \mathrm{Q}_{\mathrm{T}}$, and $\mathrm{n}_{\mathrm{i}}$ are notations used in Reference 6 . The formulas used in this section also come from Reference 6 .

**For example, $P_{i}$ may result from $P_{1}$ or $P_{2}$ where $P_{1}$ and $P_{2}$ are human errors. Thus $P_{i}=P_{1}+P_{2}-P_{1} P_{2}$. Or $P_{i}$ may be the joint probability of $P_{1}$ and $P_{2}$, where $P_{1}$ is an error and $\mathrm{P}_{2}$ is an equipment defect or some other factor which, when it occurs, sets up a potential failure condition only if a human error $\left(P_{1}\right)$ also is made. Thus $\mathrm{P}_{\mathbf{i}}=\mathrm{P}_{1} \mathrm{P}_{2}$.

The use of the exponent $n_{i}$ assumes independence of the $n_{i}$ operations. When this assumption would result in an unacceptable inaccuracy in a probability estimate, then the exponent should be dropped, and the calculation of $F_{i} P_{i}$ should take the nature of the dependence into account as is done in the example in Chapter III. Often, however, independence of certain behaviors can be assumed even when it is known that the assumption is incorrect. In such cases, it is judged that the resultant calculation of $F_{j} P_{i}$ is sufficiently accurate for the purpose at hand. $\dagger+$ There may be a different class, $j$, of failure conditions. Then $Q_{j}=1-\left(1-F_{j} P_{j}\right)^{n_{j}}$. The probability of one or more independent failures in either or both classes $(i$ and $j)$ is given as $Q_{i j}=\left(1-Q_{i}\right)\left(1-Q_{j}\right)$.
} 
In determining the effects of an error, class of errors, or group of errors on the system, two major kinds of information are needed that usually require inputs from other specialists such as reliability or project engineers and operations researchers. The first is the probability, independent of the motivation of the operator, that certain procedures will or can be followed. The second is the effect of failure in a human operation on equipment-failure rates or system effectiveness. The first probability enters into the calculation of $P_{i}$ and the second factor is $\mathrm{F}_{1}$.

In the hypothetical example used in this report, we assume a pilot must receive a message while in his aircraft and that the aircraft might either be airborne or on the ground. Error rates have to be estimated for both situations. If one wishes to determine the eflect of errors in these procedures upon system effectiveness, it is necessary to estimate the probablilty that the procedures will be done on the ground versus in the air. This type of estimation is not normally done by a human-factors specialist but can be, if he is also functioning as an operations researcher. This dual functioning is sometimes the case at Eandia Corporation.

Another estimate which should be made is the probability that a procedure can be done at all or within the required time period. In the above example, there might be environmental conditions that would preclude the possibility of the airborne pilot being able to hear a message. This type of estimate is also not normally done by a human-factors specialist. Meister (Reference 13) has pointed out that failure to consider this type of probability can lead to a gross over-estimation of system reliability. He lists five categories of "nonoperator-type discrepancies occurring insystem performance":

1. nonavailability or inadcquacy of required equipment, personne1, or technical data

2. inadequacy of procedures or technical data

3. improper personnel utilization

4. failure (not due to the operator himself) to perform required preventive maintenance or setup procedures and

5. inadequate system organization.

He states that analysis of some missile systems shows that nonoperator-type discrepancies have been almost eight times as numerous as errors which can be ascribed directly to operator accuracy. Thus, it is apparent that having reliable equipment and reliable operators does not guarantee a reliable system. Human errors in support functions of a system may account for a major source of system degradation. Therefore, the human-reliability analysis either must deal with these other sources of human error or it must restrict itself to human error which can be ascribed directly to the operators who perform system tasks. If such a restriction is made, it should be clearly stated in the analysis. 
Having estimated the probability that a procedure can be done, it is still necessary to determine the effect of an error in the pilot's performance of the procedures upon the failure rate of the system or subsystem in question. If the pilot incorrectly performs the procedures on the first trial and this failure means that his mission will inevitably fail, then an error in pilot performance is tantamount to system failure: $F_{i}$ equals 1.0 . This simple situation is not usually.found. Under certain circumstances, the pilot may have a chance to correct his error. Therefore the system failure rate related to this pilot error becomes a function of the probability that he will make the initial error and fail to correct it, and the probability of each of the circumstances. Conventional probability theory is used in writing these equations, which can be quite complicated. (Table IV and Figures 1 and 2 in Chapter III are memonic aids for setting down the proper equations.) To make sure that every contingency is included in the model, all possible alternatives must be considered. Situation diagrams which show the possible procedural paths are particularly helpful. (Figure 1 is one type of situation diagram. Figure 2 is a more specific type.) To avoid very long and complicated equations, it is usually easier to use several shorter equations which represent part-system failures. Thus, total system failure would be some combination of these part-system failures.

Chapter III continues the hypothetical example found in Chapter II in order to illustrate how one estimates the effects of human error on a man-machine system.

Recommend Changes to System and Calculate New System Failure Rate

A logical final step in applying THERP to a man-machine system is to recommend changes, if necessary, to reduce the system or subsystem failure rate to an acceptable level and then to calculate the new failure rate which results from the estimated effects of the recommended changes. The difference between the old and new failure rates can then be balanced against the various costs of changing the system. As is usual in human-factors studies, the recommended changes can take the form of modifications to equipment, training, personnel selection, or operating procedures. *

This final step provides a common sense approach to a decision on what operations should be changed. The approach consists of ranking all $Q_{i}$ values in descending order. The $Q_{i}$ value reflects the extent of system degradation resulting from the operation in question rather than merely the error rate for that operation. Ordinarily, then, operations with the highest $Q_{i}$ values would receive the most attention in looking for ways to increase system effectiveness. Whenever a change is considered, the related $P_{i}$ values are adjusted appropriately and $Q_{i}$.

\footnotetext{
*Decisions on what changes to recommend can be aided by the use of a system of error classification devised by L. W. Rook, Jr. (Reference 6). This classification scheme subdivides human error into categories which are both manageable and suggestive of the corrective action to be taken. The classification should be especially helpful to those not formally trained in human-factors technology.
} 
and $Q_{T}$ are recomputed. Changes are made until $Q_{T}$ has reached an acceptable value. As this progress is made in the iterative process of changing the system and adjusting $P_{i}$ values, cost estimates of the various changes can be obtained for comparison with the gains expected from the proposed changes.

One caution must be observed when interpreting the rank order of $Q_{i}$ 's. When a particular $Q_{i}$ has an estimated low rank, it is often tempting to omit it from further consideration, especially if available time and money limit the changes which can be made to a man-machine system at an advanced stage of development. But in the case of military man-machine systems, perhaps it should be assumed that the potential enemy is also capable of making his own analytical stidy. The potential enemy, especially if he would initiate an attack, may well evaluate lowprobability events or events assigned low importance quite differently than the designer of a retaliatory man-machine system. One shöild be cautluts, therefore, in dcciding which $Q_{1}$ 's need nu further conjideration. This caution should be selfevident, but events in our recient and past history have made it painfully apparent that errors in judgment have been made in this regard. In terms of THERP, the above consideration of the reaction of an upponent can enter into the cstimation of each $F_{i}$. 
CHAPTER II

A PROCEDURE AND SOME ASSUMPTIONS FOR ESTIMATING ERROR RATES

Introduction

This chapter presents a hypothetical example to illustrate the way in which Sandia Corporation human-factors specialists have used the AIR Data Store and other sources of human-error-rate data and have made certain gross behavioral assumptions in order to estimate human-error rates in the context of a man-machine syslem. It is reiterated here that the approach used is strictly an empirical one: if it enables us to make predictions sufficiently accurate for the purpose at hand, we use it. However, in view of the obvious gross generalizations we must sometimes make, we are far from being complacent about. the error-rate data that we use in our system reliability equations (see Chapter III). But we have reliability problems to solve and we use this data. We feel that using it is better than doing nothing, thereby either (1) forcing engineers or others not trained in human-factors technology to make their own estimates of human reliability, or (2) allowing system reliability equations to continue, as most do, to assume no degradation resulting from the human element.

\section{Use of. AIR Data}

In order to illustrate the way in which human-factors specialists can use the AIR Data Store, the following hypothetical example is given. Assume that a radio operator must contact pilots by voice radio and communicate to them a five-digit number which he has never seen before. The number is written on a special form, and we assume that the number has been written correctly. The rest of the message is routine and is "second nature". We therefore decide that the only significant source of error is the operator's voicing of the number over the radio.*

If we have no operational data, we can turn to the AIR Data Store and break down this task into its aspects and components as defined in Chapter $I$. We then turn first to the "labeling" component (Table I in Chapter I) as the input component. If we are interested in the time required for the operator to read the number to himself (i.e., the time for the input aspect), we take the base time of 0.20 and add it to the times listed opposite the following dimensions: 4-5 digits, clear and concise legibility (assuming the number is typed rather than handwritten),

*As stated in Chapter I, this restriction in the human operations to be conAs stated in Chapter $I$, this restriction in the human operations to be con-
sidered in the reliability analysis must be made with considerable caution or the results of the analysis could be misleading. 
and 1/8-inch high type. The result is 0.69 seconds. If we are interested in the probability that this input component will not lead to a reading error, take the product of $0.9992,0.9999$, and 0.9994 . This product, $0.9985, *$ is the estimate for the operator correctly reading the number to himself once. Although we have no directly relevant evidence, we hypothesize that an immediate repetition of the number by a radio operator will not significantly reduce the estimated error probability of 0.0015 . This hypothesis is based on two assumptions on nonindependence of behavioral acts. First, the operator will probably louk at the number only once even though he must repeat the number. Second, even if he looks at the number when he repeats the transmission, he will have a strong tendency to "see" the original incorrect number he read and spoke on his first transmission. This perceptual error grows out of the operator's initial reading (or input) error being reinforced by his immediate speaking of the Lncurlect numbcr (output error).

Next are the mediating processes, if any. For this step in the radio operator's procedure, we will assume that there are no requirements for long or shortterm memnry, for decision-making, or other compunents of thought subject to significant errnr. Consequently, mediating aspects can be ignored tor this scep.

To consider the output or response aspects, we turn to the page titled "Speaking" in the AIR Data Store (see Table II below). To the base time, 0.10 second, we add $0.45,0$, and 0.25 second to obtain 0.80 second. Adding to $2 \times 0.69$ second (to account for two readings), we obtain 2.18 (rounded to 2.2 seconds), the estimated time for an operator to read off a five-digit number twice. ** To round out the success prubability estimate for this step, we take the product of the values 0.9998 (for 5-10 numbers), 0.9999 (two or more repetitions), and 0.9997 (unfamiliar message using common language) to obtain 0.9994 . The product of 0.9985 , the input success probability (two repetitions), and 0.9994 , the output success probability, is 0.9979 , which we would ordinarily round to 0.998 . Thus, the estimated error rate for this step in the operator's procedure is 0.002 .

\footnotetext{
*For numbers as large as 0.99 , an approximation is suitable. Simply take the complements of each success probability figure, add them, and take the complement of the result. Thus, $1-[(1-0.9992)+(1-0.9999)+(1-0.9994)]=0.9985$. This approximation is handy inasmuch as the arithmetic manipulations can be done in one's head (with a predictable error rate, one hastens to add).

** We would know that this estimate is unrealistic inasmuch as a radio operator should pace his delivery so that the digits are spoken at a rate between 1 and 2 digits per second. So we would derive our own time estimates from other souce data. This example illustrates that one cannot apply the figures from the Data Store blindly. At Sandia, we have not needed to use the time data from the AIR Data Store because we have been able to obtain operational data or we have been able to take time measurements on eimulated operational tasks.
} 
TABLE II

Time and Success Probability Estimates for Output Component

"Speaking." (Taken from Reference 2)

BASE TIME $=0.10$ seconds

Time added Success probability (seconds) figure

$\begin{array}{ll}0 . & 0.9999 \\ 0.10 & 0.9999 \\ 0.45 & 0.9998 \\ 1.00 \text { or more } & 0.9996\end{array}$

0

0 (see

0.9998

0 above)

0.9999

0.9999

0

0.9999

0.10

0.9998

0.25

0.9997

0.40

0.9995

\section{SPEAKING}

1. Number of words or numbers (including repetitions)
a. One
b. $2-5$
c. $5-10$
d. More than 10

2. Number of repetitions
a. None
b. One
c. Two or more

3. Nature of message
a Familiar message using common language
b. Familiar message using uncommon language
c. Unfamiliar message using common language
c. Unfamiliar message using uncommon language

An Assumption for Monitored Behavior

Suppose, now, that the .002 error rate above were unacceptable (as determined by procedures described in Chapter III). Obviously, if two operators are used for this task, one to monitor the other, the task reliability should be improved. But by how much? If we square the .002 error rate, we have made the assumption of independence of behavior of the two operators. We know from studies in vigilance and inspection efficiency that a monitor of another's behavior does not ordinarily demonstrate such accuracy--in fact, far from it. Apparently, the monitor consciously or unconsciously assumes that the behavior he is monitoring has been done correctly. We therefore use a more conservative estimate of the error rate of the monitor and assign to him a .15 probability of not detecting an error made by the first operator. This figure is based upon an average inspection error rate taken from a number of studies of inspector accuracy in industrial assembly line situations, some of which are reported in Reference 14. If inspection errors are considered where operator errors are infrequent (like the .002 error rate above), then the assumed 15-percent inaccuracy of an inspector appears to be a reasonable gross estimate. Sandia 
Corporation reliability evaluations have assumed that a monitor of another man's work has only an approximate .85 probability of recognizing the latter's errors if these errors occur infrequently. Lacking any better data, this same 15-percent error rate is applied to the second operator (monitor) in our hypothetical example. Thus, the estimated probability that the wrong code will be transmitted and go undetected by both radio operators is $.002 \times .15=.0003$.

\section{An Assumption for Self-Correction of Errors}

It is apparent that there are many situations in which a man's accuracy in a task is dependerit upon errors he has just committed. That is, if a man makes an error, and then has to correce hls mlyluke, he niay have a erontor or 198ser. probability of repeating the same mistake than he did of making the mistake in the first place. In order to illustrate how Sandia Corporation human-factors specialists have handled the situation, it is convenient to expand our hypothetical example to include the pilot's hypothesized role.

We will assume that the pilot must copy down the code transmitted to him by voice while he is simultaneously engaged in other activities. We will further assume that it is important for him to get it correctly the first time because he is not supposed to acknowledge receipt of the code. Finally, we will assume that there is a good possibility that the pilot will not be in a position to ask the radio operator for a repeat of the code if he is unable to copy it down correctly the first time. Thus, it is reasonable to believe under such conditions that if the pilot is unable to translate the code because it is not the correct code, then he will have a greater probability of incorrectly copying down the code the second time he hears it, assuming that conditions permit him a second try. But if wc shanged the above conditions so that there were no particular time pressures or other stimuli leading to a high degree of pilot tenseness, then a different assumption would be justified. In facl, giveu very good conditione, one might reasnnably assume that the error rate for the second trial of the code reception task should be lower than the error rate for the first trial. And, in fact, some laboratory studies have indicated that a moderate degree of laboratory stress actually enhances accuracy in a task.* But it must not be forgotten that laboratory stress is not the same as operational stress. More is said on this subject under the next topic.

In the hypothesized example above, Sandia human-factors specialists would normally assume that the probability of incorrect code reception for the pilot's second attempt (assuming such an attempt was possible) would be double the estimated

\footnotetext{
*The validity of this statement is partly a function of the nature of the task. Reference 15 states, "If simple conditioning and complex problem solving are thought of as being at opposite ends of a continuum of mental processes, one can postulate that stress effects are facilitative for lower-end processes and detrimental for higher-end processes. In nearly all studies involving simple conditionilig, stress or anxicty was found to speed the acquisition of the conditioned response and to retard its extinction. On the other hand, most studies involving highter mental processes, such as problem-solving, thinking, discriminative learning, etc., have found that stress impairs performance."
} 
error rate for his first attempt. This assumption is stated as follows: if a man has $X$ probability of error for an important time-critical task on which errors occur infrequently, then his probability of making an error on Trial 2 (after he has made an error on Trial 1) is $2 \mathrm{X}$, for Trial 3 (given errors on Trials 1 and 2) it is 4X, for Trial 4 (given errors on Trials 1, 2, and 3) it is $8 \mathrm{X}$, and so on, until the limiting condition of a 1.0 error probability is reached. This continued doubling of his original error rate reflects the increased probability of continued error resulting from operator tenseness that occurs when it is recognized that errors are highly undesirable and that such errors might be irreversible.

To illustrate a situation where this doubling of the error rate would not be justified, let us return to the voice communication operator, and assume that he has made an error in transmitting the code and that his co-worker (i.e., the monitor) has caught this error and brought it to his attention. Due to the relatively non-stressful working conditions in a voice communication center, we would not double the voice communication operator's estimated .002 error rate of Trial 1 to obtain an estimated error rate for Trial 2. We would figure that the sma11 amount of stress or concern that the operator felt as a result of his error on Trial 1 would probably be compensated for on Trial 2 by his greater attention and care taken in his repeat of the code. Therefore, we would normally use the same error rate for both trials so that his cumulative error rate at the end of Trial 2 would be .002 squared, or $4 \times 10^{-6}$. Thus, although we wouldn't necessarily believe that the two behaviors were really independent, we would make the assumption that squaring the error rate wouldn't introduce any important decrease in the accuracy of our estimate of the cumulative error rate.

An Assumption for Behavior Under High-Stress Conditions

There is a theoretical point where a man's ability to perform a task suddenly drops off markedly. This point is a function of many factors--fatigue, training, worry, fright, human engineering of equipment, the task itself, and so on. In everyday language, we would 'say that the man's breaking point has been exceeded. T.t is well known that different men have different breaking points and that the breaking point for any one man will vary from time to time.

Although many studies have been made of the reaction of military personnel to various kinds of stress-provoking situations, very little work in guantifying the degradation of human performance under operational stress has been done.* There are situations in which it is obvious that human performance should not be considered to be occurring under normal operating conditions. Assume, for example, that our hypothetical pilot were listening to and writing down a code being transmitted to him when suddenly a barrage of anti-aircraft fire begins to shake up the aircraft. Even though he had been told that he must copy down the code correctly

* Reference 15 contains 396 abstracts from the literature on stress. Nearly all of these deal with artificial stress. 
the first time it is received, it seems ridiculous to assume that his error rate under normal conditions would apply now. It is reasonable to assume that his error rate under this high-stress condition would be considerably higher than his normal error rate. But how much higher? This is the question this section of the report discusses.

The importance to system reliability estimates of estimating human-erxor rates for tasks done under high-stress conditions is, of course, a direct function of both the probability of occurrence of these conditions and the value to the system of accurate human performance under these conditions. As stated in Chapter $I$, the second factor must not be overlooked. If a great deal of reliance is being placed upon accurate human performance under high-stress conditions to compensate for system perturbations, then it seems desirable to attempt to estimate, even by analogy, the degradation of this performance under these conditions.

It is well to reiterate that behavior degradation under artificial (laboratory or simulated) stress conditions may be misleading since there are real diffcrences between the effects of stress under artificial and operational conditions. In the latter situations, the game is for keeps, and this does seem to make d difference.* For example, physiological indices of stress obtained by flight surgeons on the X15 project indicate considerably greater homeostatic deviations (in terms of physiological measures) from normal under conditions of operational stress than under conditions of artificial stress. In flight, altitude, and other types of simulators, the normal pulse rate of 72 beats per minute of X15 pilots did in fact increase to about 110, for this was a stressful situation even though it was an artificial (nonoperational) situation. But their pulse rates under stressful operational conditions (such as drop-off from the mother aircraft or the loss of a windshield in flight) climbed to as much as 160 beats per minute. Therefore, flight surgeons have cautioned that behavior degradation (again in terms of physiological measures) under conditions of artificial stress may be a very conservative estimate of behavior degradation under conditions of high operational stress.

When trying to predict error rates under high-stress conditions, there are three factors which make such predictions tenuous. First, most of the available data have been taken under artificial conditions. Second, 1t ls well known that a person's physiological degradation under stress conditions does not neccssarily reflect a similar degradation in task behaviors. Some people, at least for a certain period of time, can maintain accurate task behavior even though they are doing so at a considerable physiological cost. Third, in most operational

* In landmark World War II study entitled Men Under Stress (Reference 16), the two psychiatrist investlgators concluded that for the vast majority of flight personnel, "the only valid test for endurance of combat is combat itself." 
situations, time-sharing of tasks is done. Under high-stress conditions, an operator may choose to attempt to maintain his normal accuracy in one or a few tasks at the expense of accuracy on other tasks.*

All of the above factors mitigate against accurate prediction of human-error rates under high-stress conditions. Nevertheless, Sandia human-factors specialists have felt it necessary to make the best estimates they can. The error rates they have used to date are based on error rates in tasks analogous to critical behaviors of Strategic Air Command (SAC) pilot's in an emergency situation in military aircraft as defined by investigators from AIR (Reference 18). These investigators defined a critical incident as a specific instance in which a crew member is called upon to handle an emergency situation. Reference 18 lists twenty-two different types of emergencies, including such conditions as complete or partial power loss, brake failures, radio equipment malfunction, and so on. In one AIR study it was found that 16 percent of all critical behaviors (e.g., behaviors which could either result in coping or not coping adequately with the emergency situation) of aircraft commanders, co-pilots, and flight engineers under emergency conditions in B-50 aircraft were ineffective. A further analysis of their data revealed that this 16 percent figure also applied to the aircraft commanders alone. The aircraft commanders had an average of about 3,000 hours flying time and averaged 885 hours in the B-50. Thus, these were well-trained personnel. At Sandia we attempted to break down the twenty-two emergency conditions into those judged to be the most and the least stress-provoking. It is interesting to note that both categories still yielded approximately 16 percent ineffective behaviors. Either the twenty-two emergency conditions are comparable in terms of generating human errors or we made an erroneous lreakdown. Assuming the former explanation is more nearly correct, generalizing the above 16 percent figure to other operational situations judged to be analogous to responding to aircraft emergencies may, at least suggest a basis for estimating error rates under high-stress conditions.

This treatment of high-stress is essentially the one used at Sandia. An estimate of 10 to 20 percent error rate for pilot tasks analogous to the critical behaviors identified in the AIR study is felt to be a reasonable and expedient approximation until better data can be obtained. Because of less stringent timesharing requirements, an estimate of 5 to 15 percent has been used for high-stress error rates for in-flight tasks performed by aircrewmen other than the pilot. A range is used as a reflection of the grossness of the estimate. Thus, in the example of our hypothetical pilot, we would assign him an error-rate of 10 to 20 percent for code reception under high-stress conditions such as being shot at. In reliability

"Some British investigators studied this phenomenon of the "lowered standard" under conditions of fatigue and stress. It was concluded (Reference 17) that within the skill being investigated, "There are always two (discrimination) thresholds-one a measure of what the observer can do, and the other of what is trcated as worth doing. These can, and constantly do, vary quite independently. At the beginning of exercise they normally approximate to the same value though they are never quite identical. With continued exercise, or under a variety of other conditions, they diverge more and more. The threshold of discrimination-what the operator can do--is little affected, except in extreme cases; the threshold of indifference-what is treated as worth doing--may rise to double, treble, or quadruple its original value.... The operator may know nothing about it. He may assert that his skill is exactly as it was, and if he is stopped and his threshold of discrimination measured he may appear to be right. For a genuine measure of his skill he needs to have both these thresholds determined with the operation itself." 
analyses where it has been necessary to make an estimate of behavior degradation under high-stress conditions, we feel that if our estimates of error rates for any given application are incorrect, they can probably be assumed to be on the average no more than an order of magnitude too high. We are unable to make a comparable estimate of the extent to which the estimate might be an underestimation of the operational high-stress error rate.

The question has arisen in past reliability analyses of the effect of an error in a high-stress task situation upon the operator's attempt to correct that error, assuming conditions allow such an attempt. In such cases we have kept the same assumption that we applied to tasks done under normal operational conditions. We have assumed that each repetition of a high-stress task after an error has been made in its immediately preceding performance will double the preceding error rate until the error probability of the task is 1.0. This assumption is certainly open to some question, but it appears to be a better assumption than one of independence of behavior between trials. Most behavioral scientists state that stress is a person's internal response to his perception of events, and large individual differrences can be expected in responding to an error in a critical task. Thus, some people rapidly deteriorate in a situation where a high error rate occurs.

Furthermore, the more severe the stress, the sooner the point is reached at which many men begin to respond to their errors with more and more errors. In short, under highly stressful conditions, an individual may become extremely likely to continue to perform erratically with many errors. Thus, under severe stress our hypothetical pilot, having made a code reception error on Trial 1 , may become so errorsusceptible that his ability rapidly decreases to zero. Or, on the other hand, he may so concentrate on the reception task that his flying performance may be degraded to a danger point. In any event, it will be noted that doubling of large error rates does force the error-rate to unity after a relatively small number of trials. Implicit in this procedure, therefore, is the realistic concept that behavior will rapidly break down completely under high-stress if errors continue to be made."

It is apparent that the esllmales of lighi-stress crror rater are sertainly very gross. They depend upon several judgments made by the analyst, not the least of which is his judgment that behavior in a particular high-stress task is analogous to a SAC pilot's behavior in responding to an operational emergency. It is in this high-stress area that relevant research on operational error-rates is most urgently needed. $* *$

\footnotetext{
*For example, given an initial high-stress error rate of 0.10 , it will take approximately five trials (original error plus four incorrect attempts to correct the error) for the error rate to reach 1.00 . Note, however, that the probability of reaching the 1.00 error rate is approximately $.10 \times .20 \times .40 \times .80 \times 1.00 \approx .0064$, which we would approximate as .01 in view of the grossness of our estimates. This estimate further assumes the occurrence of the high-stress situation and its continuation for the entire corrective sequence of actions. (It will be noted that the fifth term in the above equation is 1.00 rather than $2 \times .80$ or 1.60 . A probability of over 1.0 is meaningless.)
}

${ }^{*} *$ L. W. Rook, Jr., Sandia Corporation, is currently conducting a study in which he is attempting to quantitatively relate error rates to varying levels of operational stress. 


\title{
CHAPTER III
}

A PROCEDURE TO ESTIMATE THE EFFECTS OF HUMAN ERRORS ON A MAN-MACHINE SYSTEM

\author{
Introduction
}

This chapter continues the hypothetical example begun in Chapter II to show how Sandia Corporation human-factors specialists estimate the effects of human errors on a man-machine system or any part of it. The general procedure is described in Chapter I. This procedure involves the construction of an appropriate mathematical model and the assignment of $Q_{i}$ values, that is, the probability of one or more failure conditions of the system, or that part of the system being evaluated, existing as a result of ciass $i$ errors occuring in $n_{i}$ operations. As stated in Chapter $I, Q_{i}$ has three major determinants. First, there is the error rate itself. Second, there is the probability that a human error will result in a failure to perform some procedure. Third, there is the probability that this procedure is supposed to be used and can be used in the operation of the system. The example which follows attempts to illustrate how the relevant probability figures are derived and used in arriving at an estimate of the effect of human errors on the system.

\section{A Hypothetical Operational Situation}

\section{Assumed Operational Sequence}

Over a voice radio circuit monitored by the pilots of single-place aircraft, a voice communicator reads a five-digit code aloud twice while being checked by an assistant communicator (monitor). This is an open transmission; the pilots do not break radio stlence. Each pilot in his aircraft, which may be either on the ground or airborne, writes down the code as he is listening to it, unless he is forced to time-share this task with other tasks which also demand considerable attention. He is not allowed to ask for a repeat of the code at this time but must first try to translate the code he has copied down. The translation of the code is done in some specific manner which need not be hypothesized here. His answer is either obviously correct or obviously wrong, and this is immediately apparent to him. If he has obtained an incorrect answer, then he will immediately repeat the translation task, inasmuch as he might have made an error. If this repetition does not provide the correct answer, then he will attempt to contact the voice communicator and obtain a repeat of the code. If this attempt is unsuccessful, then the mission is a failure. If the attempt is successful, then he will again perform the code translation task, twice if necessary. If the answer is still incorrect, then the mission is arbitrarily considered to be a failure. (This last limitation is made to provide an 
ending point for the hypothetical sequence.) The mission may also fail because of events not under the control of the pilot or voice communicator.

\section{Major Assumptions}

1. The five-digit code written on a paper from which the voice communication operator reads is the correct code.

2. If the monitor correctly or incorrectly tells the voice communicator that he made a communication error, for the purpose of simplicity it is assumed that the end result will always be the correct communication. Thus, a communication error will only result if an error gets by both communicators.

3. 'l'he pilot who received the code via voice radio has received the usual training including simulated practice in all the tasks required of him.

4. Wartime conditions have just been initiated, and it is possible that all personnel on the ground may come under enemy attack. (This assumption sets the basic level of stress.)

5. The correct reception and translation of the code is necessary for the pilot's completion of his mission.

6. Certain operational circumstances might occur that make it impossible for some of the required tasks to be performed at all or within some allowable time period. (All tasks are possible unless otherwise noted.)

7. A11 equipment released for operational use is 100-percent reliable.*

8. Whatever conditions (high-stress/nurmal-medium-stress/normal-stress or air/ground operations) hold for the pilot or voice communicators on their first trials on code tasks also hold for the remainder of their operations.

9. The communication channel is either blocked completely or open completely although the signal-to-noise ratio will vary. If the channel is blocked, the mission is a failure.

*This assumption, made to simplify the example, actually should not be too unreasonable in a real situation because. any equipment unreliability associated with the tasks as described should be so small in comparison with the human-error rates or degrading effects of certain operational circumstances that the effect of the equipment unreliability would probably be masked. 
10. The voice communicators operate under normal-stress conditions at all times.

11. No malicious errors are made.

\section{Situation Diagram}

Figure 1 is a situation diagram (not a flow diagram) which represents the operational sequence described earlier. The Greek letters and related numbers stand for the probabilities of events not under the control of the communicators and pilot. The capital English letters* and related figures stand for the rates for uncaught errors (by himself) of the communicator, assistant communicator, or pilot. The meaning of each symbol is described in Table III below.

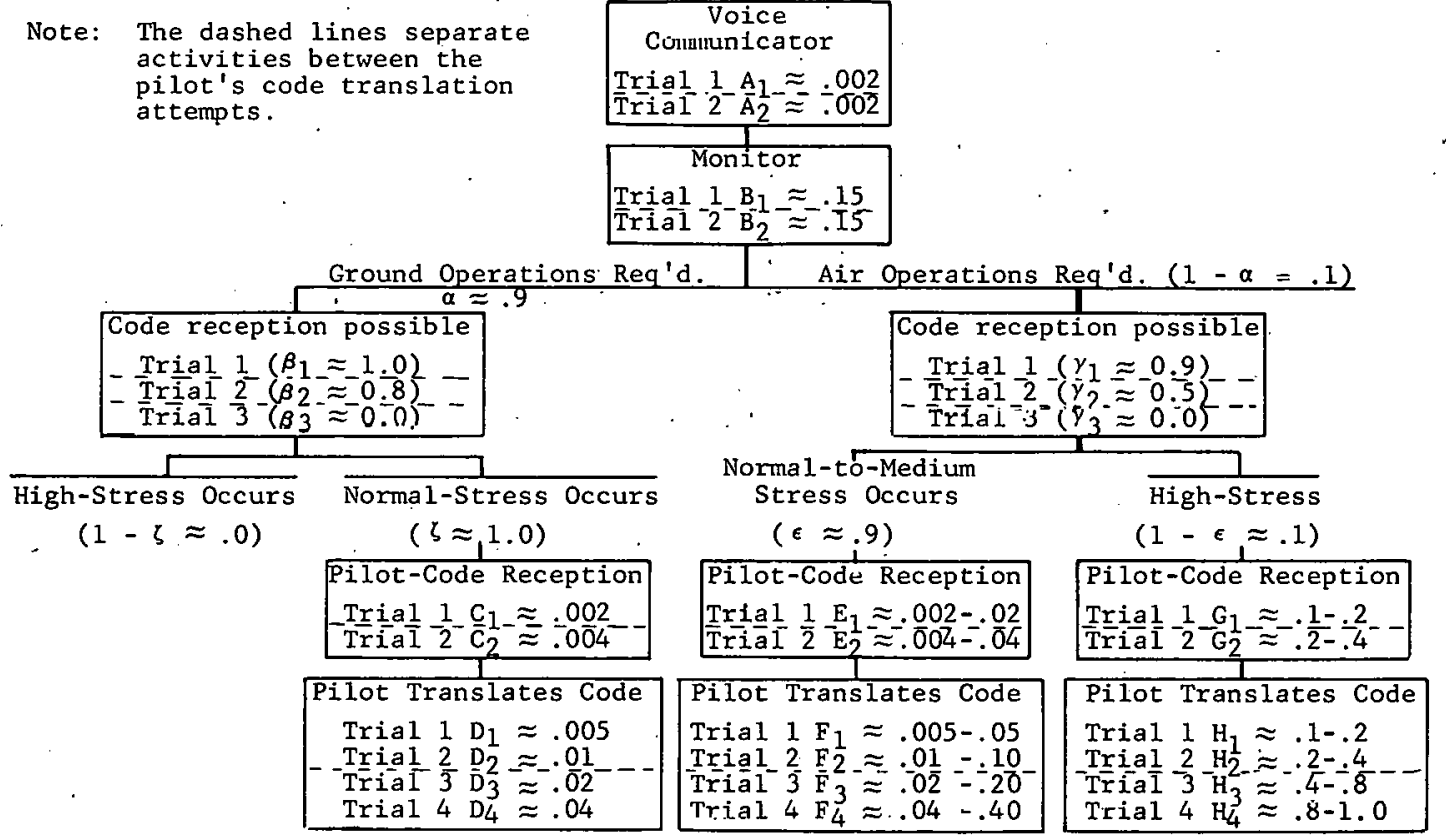

Figure 1. Situation Diagram of Hypothetical Military Sequence

\section{Probability Estimates}

Al1 probability estimates in Table III are hypothetical approximations.

\footnotetext{
*At Sandia Corporation, capital English letters are conventionally used for error rates or failure rates. Small English letters are conventionally used for success probabilities.
} 
Assistant communicator fails to catch error of voice

communicator, or erroneously concludes that communicator has made an error, Trial 1

Pilot on ground copies down transmitted code incorrectly, Trial 1

Same, Trial 2

Pilot on ground translates code incorrectly Trial 1

Pilot in air, normal-to-medium stress condition, copies down transmitted -code incorrectly, Trial 1

Pilot in air, normal-to-medium stress conditions, translates code incorrectly, Trial 1 . 
The hypothetical system criterion being evaluated is the successful translation of the five-digit code by the pilot. The probability of system success, then, is 1 minus the probability of failing to arrive at a correct translation of the code. Naturally, to estimate the degradation resulting from human error, it must be isolated from the effects of other failure events. Thus, the system failure rate resulting from human errors equals (1) the probability of not having the other failures multiplied by (2) the probability of human failure to arrive at a correct code translation.

\section{Direct Versus Indirect Approach}

In calculating the degradation resulting from human error, one can take either of two approaches when using the exact probability equations. One can directly calculate the system degradation by appropriately combining failure probabilities or one can indirectly calculate the system degradation by appropriately combining success probabilities and then subtracting the total from unity. Either model will work, but frequently one model will be considerably more complex than the other. Usually, when a man-machine system being analyzed will yield more series events than parallel events, and when one is using the exact probability equation, it is better to use the indirect approach and calculate the success probabilities. This principle can be verified readily by reference to Table $\mathrm{IV}^{*}$.

Figure 2 on page 32 and also as a fold-out page (61) at the end of this report enables one to apply this principle in determining which approach to use for the exact equation. There are a considerable number of contingent events in this hypothetical example, including a second communication attempt in case the first attempt and subsequent activities fail to result in correct translation of the code. Thus, in this example, it is simpler to use the direct approach. However, in order to fully illustrate probability technology as applied to human. factors, both the direct and indirect approaches will be presented.

*Note in the 2-switch circuit in Table IV that the success probability series equation is $S=a b$, whereas any of the failure equations is more complex. The opposite holds for. the success probability parallel equation. Note that as the circuits contain more elements, the difference in complexity increases between the success and failure probability equations for series and parallel events.

Table IV should be helpful to those who are not very familiar with probability concepts. Such persons might well study Table IV before proceeding further in the report. 
Let $A$ and $B$ be open switches (or failure conditions) and $a$ and $b$ be closed switches (or success conditions). $F=$ failure and $S=$ success. Each cell is the product of its marginal terms, i.e., in Part I SERIES, cell $A B$ equals the product of its marginal terms $A$ and $B$.

Part 1. A 2-switch circuit
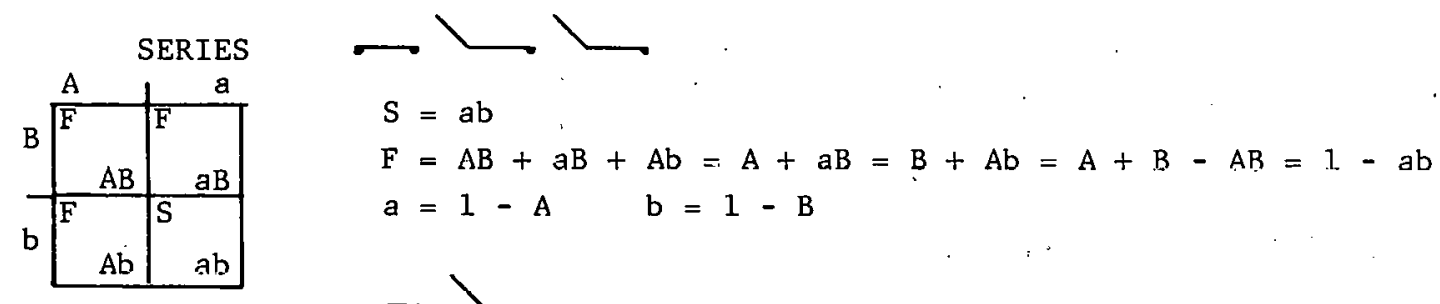

PARALLEL

\begin{tabular}{|c|c|c|}
\hline \multicolumn{2}{|c|}{$\mathrm{A}$} & a \\
\hline & $F$ & $\dot{s}$ \\
\hline$B$ & $A B$ & $\mathrm{aB}$ \\
\hline & $S$ & $S$ \\
\hline & $\mathrm{Ab}$ & $a b$ \\
\hline
\end{tabular}

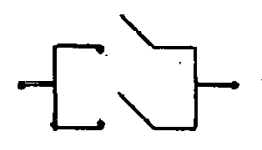

$$
\begin{aligned}
& F=A B \\
& S=A b+a B+a b=b+a B-a+A b=a+b-a b=1-A B
\end{aligned}
$$

Part 2. A 3-switch circuit

\section{SERIES}
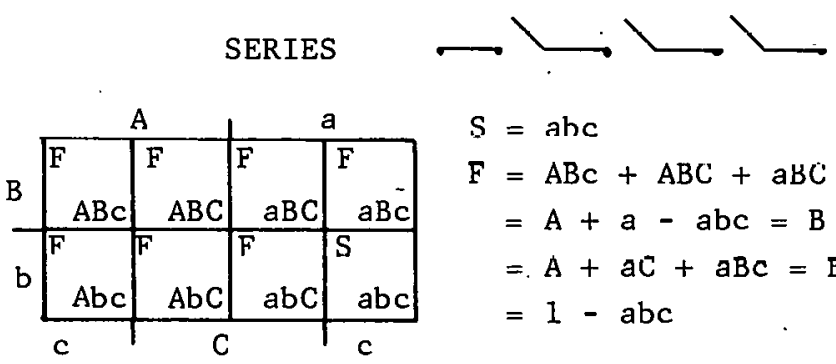

$$
S=a h c
$$

$F=A B c+A B C+a B C+a B c+A b c+A b C+a b C$

$=A+a-a b c=B+b-a b c=C+c-a b c$

$=A+a C+a B C=B+b C+A b c=C+A c+a B c$

$=1-a b c$

\begin{tabular}{|c|c|c|c|c|}
\hline & \multicolumn{2}{|c|}{$A$} & \multicolumn{2}{|c|}{ d } \\
\hline & $\bar{S}$ & $\bar{F}$ & $\bar{S}$ & $S$ \\
\hline B & $\mathrm{ABC}$ & $\mathrm{ABC}$ & $\mathrm{aBC}$ & $a B c$ \\
\hline & $S$ & $S$ & & S \\
\hline & $\mathrm{Abc}$ & $\mathrm{AbC}$ & $a b c$ & $a b c$ \\
\hline
\end{tabular}

PARALLEL

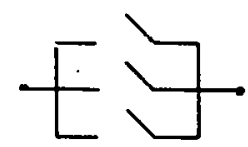

$$
\begin{aligned}
F & =A B C \\
S & =A B c+a B C+a B c+A b c+A b C+a b C+a b c \\
& =a+A-A B C=b+B-A B C=c+C-A B C \\
& =a+A b+A B c=b+a B+A B c=c+a C+A b C \\
& =1-A B C
\end{aligned}
$$


Part 3. A 4-switch circuit

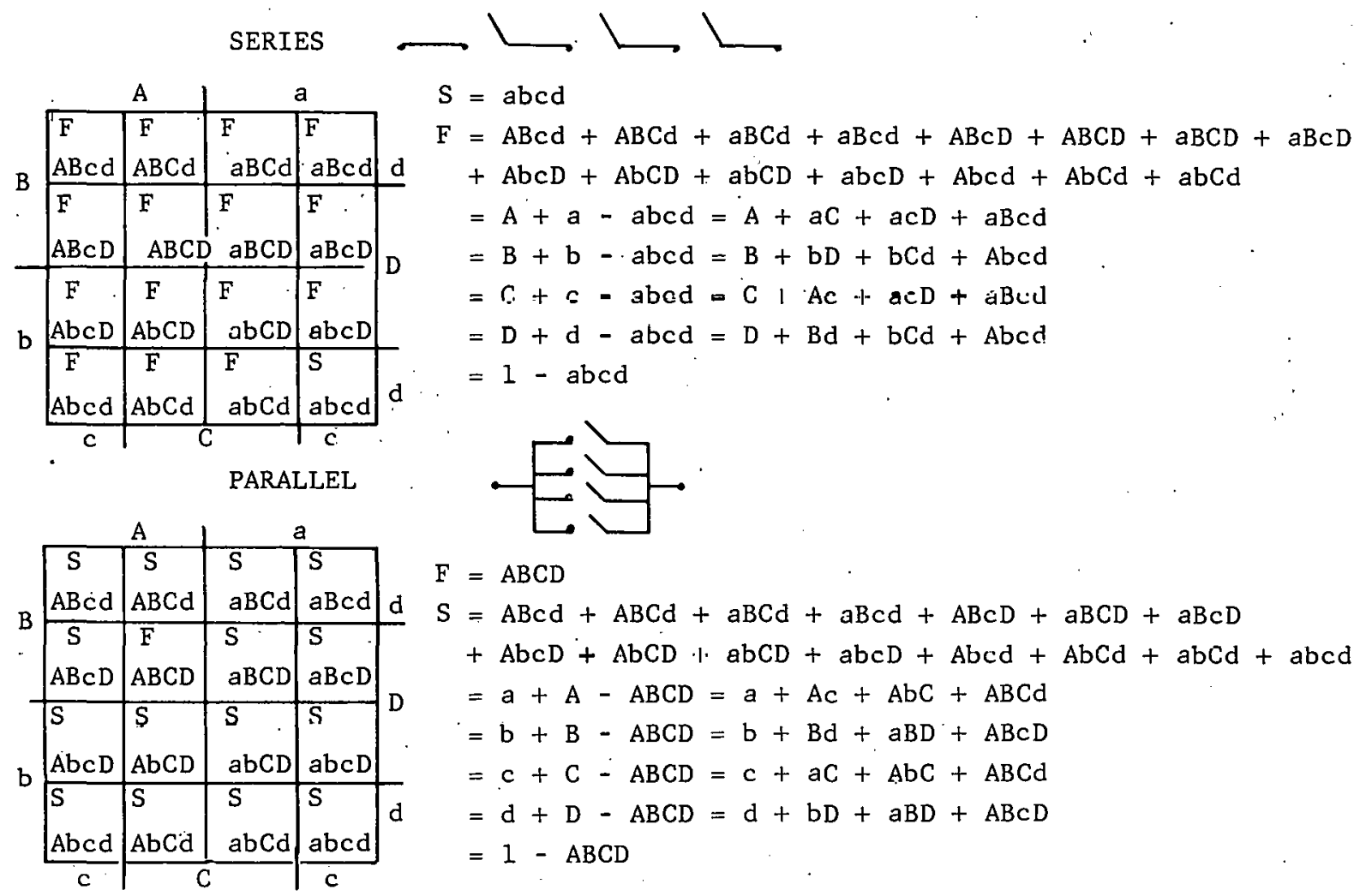

Part 4. A4-switch series parallel circuit

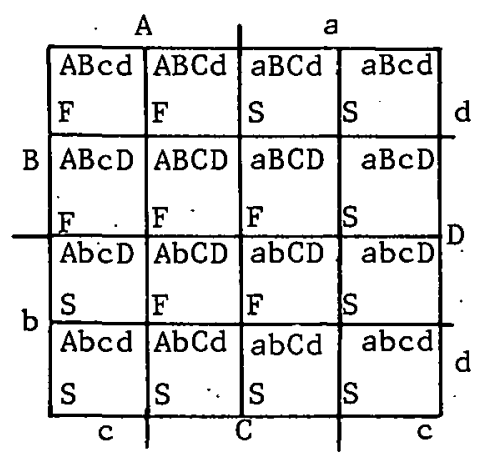

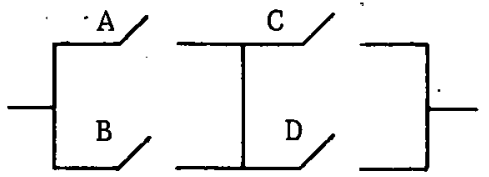

$F=A B C d+A B C d+A B C D+A B C D+a B C D+A b C D+a b C D$

$=A B+C D-A B C D$

$S=a B C d+a B c d+a B c D+A b c D+a b c D+A b c d+A b C d$

+ abCd. + abcd:

$=a c+b d-a b c d+A b c D+a B C d$ 


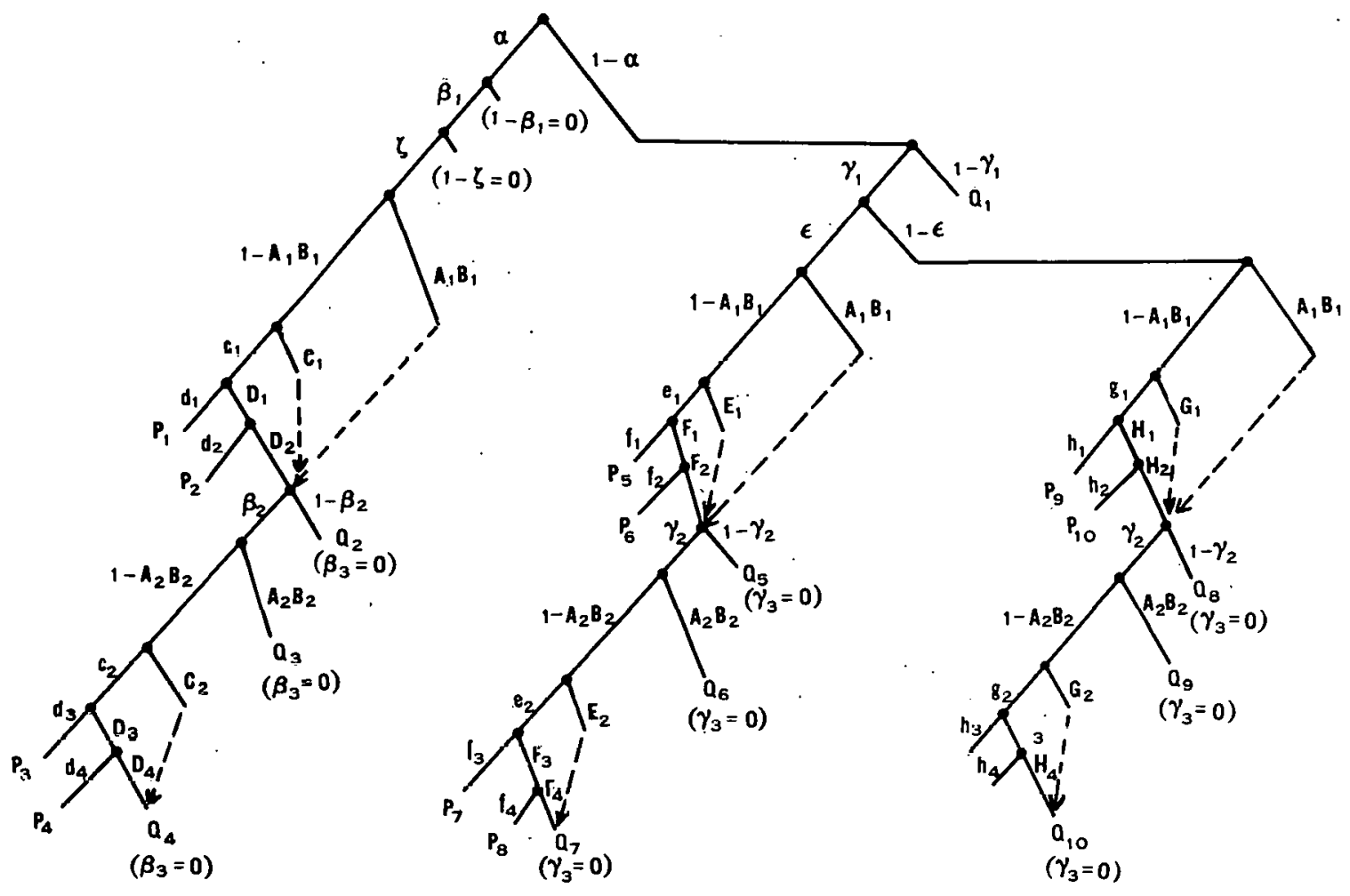

( $P=$ success. $Q=$ failure. Small English letters and Greek letters represent successes. Capital English letters represent failures.)

Figure 2. Probability tree illustrating branching technique

Figure 2 illustrates the branching technique used in the Reliability Department. at Sandia Corporation. As stated previously, small English letters represent success probabilities and capital English letters represent failure probabilities, or, in this case, error rates of tasks. $Q$ and $P$ represent, respectively, failure and success of a branch of operations. The meanings of the letters and subscripts are given in Tahle. TTT.

\section{The Indirect Approach}

The left-most branch $\left[a, \beta_{1}, \zeta,\left(1-A_{1} B_{1}\right), c_{1}, d_{1}\right]$ represents one way of successfully achieving the system criterion, i.e., correct translation. Ground operations were required; normal-stress conditions prevailed; the code was communicated correctly*; and the pilot successfully performed the reception and translation tasks the first time. Other branches of the tree can be interpreted in much the same fashion.

*We have assumed that any communicator behaviors except $A_{1} B_{1}$ lead to transmission of the correct code. If this is true, then the term $\left(1-A_{1} B_{1}\right)$ expresses the probability of successful transmission of the correct code from the point of origin. Similarly, $\left(1-A_{2} B_{2}\right)$ represents the same sequence of events for the second communication attempt. (In either case, of course, currect transmission does not guarantee correct reception.) 
The dashed lines represent shortcuts used in lieu of diagramming al1 of the failure branches. For example, the branch ending at $C_{1}$ indicates that the pilot made an error in code reception. From this point, whether or not he correctly performs the human activities associated with code translation makes no difference-he will not obtain the desired indication of a correct code translation because he has used an incorrect code. The dashed line shows that he will eventually wind up at the point where he tries for a repeat of the code.

Once the branches on the probability tree have been diagraimed, it is a simple matter to set down the equation which represents the degradation $\left(Q_{T}\right)$ resulting from the human element. This equation is given by:

$$
Q_{T}=1-\left(P_{1}+P_{2}+\cdots \cdots P_{12}\right)-Q_{1}
$$

This equation assumes independence of the branches. It is necessary to remove fallure $Q_{1}$ from the equation since we are restricting the equation to system failure due to human error. $Q_{1}$, which equals $(1-a)\left(1-\gamma_{1}\right)$, results from air operations being required but with conditions which prohibit the pilot's receiving the code. Note that $\left(1-\beta_{2}\right)$ and both $\left(1-\gamma_{2}\right)$ terms are not subtracted from $Q_{T}$ even though they are not human failures as defined in our analysis. This is because these terms can only occur if there has been some human error in the first half of each branch, i.e., prior to $\beta_{2}$ or $\gamma_{2}$. Therefore, these terms are considered to be parts of the human initiated system failure rate.

By substituting the appropriate terms, $Q_{T}$ can be further detailed and the estimated degradation calculated. Rather than introduce this lengthy equation at this point, it will be more understandable if it is developed by degrees. Consider only the left-most third of the probability tree. To express the success probability for this part of the tree, we simply set down all the paths which lead to success and ignore those which lead to failure. Thus, the probability of success, given $a$, can be written as

$$
\begin{aligned}
P_{S \mid a} & =\left(1-A_{1} B_{1}\right) c_{1}\left[d_{1}+D_{1} d_{2}+D_{1} D_{2} \beta_{2}\left(1-A_{2} B_{2}\right) c_{2}\left(d_{3}+D_{3} d_{4}\right)\right] \\
& +\left[A_{1} B_{1}+\left(1-A_{2} B_{1}\right) C_{1}\right]\left[\beta_{2}\left(1-A_{2} B_{2}\right) c_{2}\left(d_{3}+D_{3} d_{4}\right)\right]
\end{aligned}
$$

$\beta_{1}$. and $\zeta$ are omitted because they both equal 1.0. Note also that the behaviors denoted by the dashed lines (but not shown in the probability tree) are not included in the above equation. One dashed line runs from $C_{1}$ to $\beta_{2}$. Since the behaviors denoted by this dashed line equal unity, they are omitted from the equation.*

"Given $C_{1}$, no matter what the pilot does, he will not obtain the indication for a correct translation of the code. Thus, he will have to attempt recommunication of the code. The equation for this set of events, given $a$, is given as $\left(1-A_{1} B_{1}\right) C_{1}\left[d_{1} d_{2}+d_{2} D_{2}+D_{1} d_{2}+D_{2} D_{2}\right] \beta_{2}$ etc.

The expression in brackets can be written as $d_{1} d_{2}+D_{1} d_{2}+d_{1} D_{2}+D_{1} D_{2}$ or

$$
d_{2}\left(d_{1}+D_{1}\right)+D_{2}\left(d_{1}+D_{1}\right) \text { or }\left(d_{2}+D_{2}\right)\left(d_{1}+D_{1}\right) \text {. }
$$

The latter two quantities both equal unity. 
Another dashed line runs from $A_{1} B_{1}$ to $\beta_{2}$. The behaviors denoted by this dashed line also equal unity and again are omitted from the equation.*

If we wanted to obtain an estimate of system degradation, given $a$ (that is, given ground operations), the appropriate probability estimates could be substituted for the terms in the equation. Using the estimates listed earlier,

$$
\begin{aligned}
\mathrm{Q}_{\mathrm{S}}= & 1-\mathrm{r}_{\mathrm{S}} \\
= & 1-\{.9997 \times .998[.995+(.005 \times .99)+(.005 \times .01 \\
& \times .8 \times .9997 \times .996)(.98+.02 \times .96)] \\
& +[.0003+.9997 \times .002][.8 \times .9997 \times .996(.98+.02 \times .96)]\} \\
\approx & 1-.99948 \approx .00052 \approx 5 \times 10^{-4}
\end{aligned}
$$

This estimated degradation due to the human element indicates that if all of our assumptions are correct, then the hypothetical system is in good shape, given ground operations for all pilots. If our assumpliun--given $D_{3}$, then there is suffirifnt time for $d_{4}--i$ s not correct, then our esclmate of system degradation would be ton small, but probably not an important amount in an operational situation. If our assumed values for $\beta_{1}$ and $\beta_{2}$ are overestimates, then the estimated degradation of $5 \times 10^{-4}$ would be overoptimistic.

On the other hand, if our assumption that $\beta_{1}$ is 1.0 is correct, the system would still he fairly reliable even if $\beta_{2}$ were zero. That is,

*Wile practice enables one to write down the above equation directly from the probability tree (but usually with one or mure errors on thc first attempt), fewer errors are likely to be made if all of the terms in the equation are written out and then the equation is simplified by appropriate factoring. Thus, the following terms would lead to the equation above.

$$
\begin{aligned}
P_{S \mid a}= & \beta_{2} \zeta\left(1-A_{1} B_{1}\right) c_{2} d_{2}+\beta_{1} \zeta\left(1-A_{2} B_{1}\right) c_{1} D_{1} d_{2} \\
& +\beta_{1} \zeta\left(1-A_{1} B_{1}\right) c_{1} D_{2} D_{2} \beta_{2}\left(1-A_{2} B_{2}\right) c_{2} d_{3} \\
& +\beta_{1} \zeta\left(1-A_{1} B_{1}\right) c_{1} D_{2} D_{2} \beta_{2}\left(1-A_{2} B_{2}\right) c_{2} D_{3} d_{4} \\
& +\beta_{1} \zeta\left(1-A_{2} B_{1}\right) c_{2} \beta_{2}\left(1-A_{2} B_{2}\right) c_{2} d_{3} \\
& +\beta_{1} \zeta\left(1-A_{2} B_{1}\right) c_{2} \beta_{2}\left(1-A_{2} B_{2}\right) c_{2} D_{3} d_{4} \\
& +\beta_{1} \zeta A_{1} B_{1} \beta_{2}\left(1-A_{2} B_{2}\right) c_{2} d_{3} \\
& +\beta_{2} \zeta A_{1} B_{1} \beta_{2}\left(1-A_{2} B_{2}\right) c_{2} D_{3} d_{4} \\
= & \left(1-A_{1} B_{1}\right) c_{1}\left[d_{1}+D_{1} d_{2}+D_{1} D_{2} \beta_{2}\left(1-A_{2} B_{2}\right) c_{2}\left(d_{3}+D_{3} d_{4}\right)\right] \\
& +\left(1-A_{2} B_{2}\right) C_{2} \beta_{2}\left(1-A_{2} B_{2}\right) c_{2}\left(d_{3}+D_{3} d_{4}\right) \\
& +A_{2} B_{1} \beta_{2}\left(1-A_{2} B_{2}\right) c_{2}\left(d_{3}+D_{3} d_{4}\right) \\
= & \left(1-A_{1} B_{1}\right) c_{1}\left[d_{1}+D_{1} d_{2}+D_{2} D_{2} \beta_{2}\left(1-A_{2} B_{2}\right) c_{2}\left(d_{3}+D_{3} d_{4}\right)\right] \\
& +\left[A_{1} B_{1}+\left(1-A_{2} B_{1}\right) C_{1}\right]\left[\beta_{2}\left(1-A_{2} B_{2}\right) c_{2}\left(d_{3}+D_{3} d_{4}\right)\right]
\end{aligned}
$$




$$
\begin{aligned}
Q_{S|a|\left(B_{2}=0\right)} & \approx 1-\left[\left(1-A_{1} B_{1}\right) c\left(d_{1}+D_{1} d_{2}\right)\right] \\
& \approx 1-[.9997 \times .998 \times(.995+.005 \times .99)] \\
& \approx .0003+.002+.0001^{*} \\
& \approx .0024 \approx 2 \times 10^{-3}
\end{aligned}
$$

However, in some operational situations, this increase in system unreliability by a factor of four might not be acceptable.

Let us now. turn to the calculation of the estimated $Q_{T}$, the total system degradation per system resulting from human error in this hypothetical example. The following equation brings in all of the success probabilities (except the ones that equal. 1.0) in the probability tree of Figure 2. The low range, $\mathrm{Q}_{\mathrm{T}}$, is calculated first.

$$
\begin{aligned}
& \mathrm{Q}_{\mathrm{T}_{2}} \approx 1-\left[a \left\{( 1 - \mathrm { A } _ { 1 } \mathrm { B } _ { 1 } ) \mathrm { c } _ { 2 } \left[\mathrm{d}_{1}+\mathrm{D}_{1} \mathrm{~d}_{2}+\mathrm{D}_{1} \mathrm{n}_{2} \beta_{2} \cdot\left(1-\Lambda_{2} \mathrm{~B}_{2}\right) c_{2} \cdot\left(\mathrm{d}_{3}\right.\right.\right.\right. \\
& \left.\left.+D_{3} d_{4}\right)\right]+\left[A_{1} B_{1}+\left(1-A_{2} B_{2}\right) C_{1}\right]\left[B _ { 2 } ( 1 - A _ { 2 } B _ { 2 } ) c _ { 2 } \left(d_{3}\right.\right. \\
& \left.\left.\left.+D_{3} d_{4}\right)\right]\right\}+(1-a)\left[\gamma _ { 2 } \left[\epsilon \left\{( 1 - A _ { 1 } B _ { 1 } ) e _ { 1 } \left\{f_{1}+F_{1} f_{2}\right.\right.\right.\right. \\
& \left.+F_{2} F_{2} \gamma\left(1-A_{2} B_{2}\right) e_{2}\left(E_{3}+F_{3} f_{4}\right)\right\}+\left\{A_{1} B_{1}+\left(1-A_{1} B_{2}\right) E_{1}\right\} \\
& \left.\left\{\gamma_{2}\left(1-A_{2} B_{2}\right) e_{2}\left(f_{3}+F_{3} f_{4}\right)\right\}\right\} \\
& +(1-\epsilon)\left\{( 1 - A _ { 1 } B _ { 1 } ) g _ { 1 } \left\{h_{1}+H_{1} h_{2}+H_{1} H_{2} \gamma_{2}\left(1-A_{2} B_{2}\right)\right.\right. \\
& \left.x g_{2}\left(h_{3}+H_{3} h_{4}\right)\right\}+\left\{A_{2} B_{2}+\left(1-A_{1} B_{1}\right) G_{2}\right\}\left\{\gamma_{2}\left(1-A_{2} B_{2}\right) g_{2}\right. \\
& \left.\left.\left.\left.\left.x\left(h_{3}+H_{3} h_{4}\right)\right\}\right\}\right]+\left(1-\gamma_{1}\right)\right]\right] \text {. } \\
& \approx 1-[.9\{.9997 \times .998[.995+(.005 x .99)+(.005 \times .01 \times .8 \\
& \times .9997 \times .996)(.98+.02 \times .96)]+[.0003+(.9997 \times .002)] \\
& \times[.8 \times .9997 \times .996(.98+.02 \times .96)]\} \\
& +.1[.9[.9\{.9997 \times .998\{.995+(.005 \times .99)+(.005 \times .01 \\
& \times .5 \times .9997 \times .996)(.98+.02 \times .96)\}+\{.0003+(.9997 \times .002)\} \\
& \times\{.5 \times .9997 \times .996(.98+.02 \times .96)\}\}+.1\{.9997 \times .9\{.9 \\
& +(.1 \times .8)+.1 \times .2 \times .5 \times .9997 \times .8(.6+.4 \times .2)\}+\{.0003 \\
& +(.9997 \times .1)\}\{.5 \times .9997 \times .8(.6+.4 \times .2)\}\}]+.1]] \\
& \approx 1-[.9\{.9995\}+.1[.9[.9 \mid .9990\}+.1\{.9121\}]+.1]] \\
& \approx 1-.9987 \approx .0013 \approx 10^{-3}
\end{aligned}
$$

$\approx$ The low range of the estimated average total system degradation per system due to human error.

\footnotetext{
* This method of approximation was explained in Chapter II. It saves the multiplication of large numbers, and can ordinarily be used when the error rates
are in the neighborhood of 1 percent or less.
} 
The high range of $Q_{T}$ is calculated by substituting the high range of probability estimates where appropriate. This $Q_{\mathrm{T}_{2}}$ equals approximately $4 \times 10^{-3^{*}}$. Thus, $Q_{T}$, the estimated total system degradation due to human error, is between 0.1 and 0.4 percent regardless of whether one uses the high or low probability estimates from Table III. The reason for this relative insensitivity to large variations in. some error rates can be traced to the relatively. low estimated probability of the occurrence of the middle branch of the probability tree, $(1-a) \gamma_{1} \epsilon=.1 \times .9 \times .9=$ .081 , and the even lower estimated probability of the righthand branch, $(1-a) \gamma_{1}$ $(1-\epsilon)=.1 \times .9 \times .1=.009$, as compared with the estimated probability of the lefthand branch, $a \beta_{1}=.9 \times 1.0=.9$.

It should be recalled that it was necessary to subtract $(1-a)\left(1-\gamma_{1}\right)$ from the equation of system failure due to human error, to account for the fact that $(1-a)\left(1-\gamma_{1}\right)$ is not a failure due to human error as defined. If this term (which equals .01) is added back into the equation, the result, $10^{-3}+10^{-2}+10^{-2}$, shows clearly that the failure rate due to pilot and communicator human error is only about $1 / 10$ th of the total failure rate of that part of the system evaluated.

Some operational personnel object to the above type of syslem ealculation. They would prefer to estimate (1) the system degradation due to humalt error, givon $a$; (2) this degradation, given $1-a$ and $\epsilon$; and (3) this degradation, given $1-a$ and $1-\epsilon$. Their argument may be summarized as follows: "Although there may be conditions not under the control of the operator which seriously degrade system reliability, we want to make sure that if these conditions are favorable, then the operator will have a high probability of success." Without commenting on the validity of this argument, it should be apparent that the approach described herein permits these calculations.

\section{The Direct $\Lambda$ pproach}

As stated earlier, in calculatlug the cxact failure equation for the hypothetical example in this report, it is simpler to use failure probabilities rather than success probabilitica. This sertion of the report illustrates the calculations using failure probabilities and the following section shows and discusses an approximation using approximate failure probabilities.

$$
\begin{aligned}
& \mathrm{Q}_{\mathrm{T}}=a\left[\mathrm{~A}_{2} \mathrm{~B}_{2}+\left(1-\mathrm{A}_{2} \mathrm{~B}_{2}\right) \mathrm{C}_{1}+\left(1-\mathrm{A}_{1} \mathrm{~B}_{2}\right) \mathrm{c}_{2} \mathrm{D}_{2} \mathrm{D}_{2}\right] \\
& {\left[\left(1-\beta_{2}\right)+\beta_{a} A_{2} B_{a}+\beta_{a}\left(1-A_{2} B_{2}\right) C_{a}+\beta_{a}\left(1-A_{2} B_{2}\right) c_{2} D_{3} D_{4}\right]} \\
& +(1-a) \gamma_{1}\left[\epsilon\left[A_{1} B_{1}+\left(1-A_{1} B_{2}\right) E_{2}+\left(1-A_{1} B_{1}\right) e_{2} F_{1} F_{a}\right]\right. \\
& {\left[\left(1-\gamma_{2}\right)+\gamma_{2} A_{2} B_{2}+\gamma_{2}\left(1-A_{2} B_{2}\right) E_{2}+\gamma_{2}\left(1-A_{2} B_{2}\right) e_{2} F_{3} F_{4}\right]} \\
& * \quad \mathrm{Q}_{\mathrm{T}_{2}} 1-[.9 \cdot\{.9995\}+.1[.9[.9\{.9859\}+.1\{.7516\}]+.1]] \\
& \approx 1-.9962 \approx .0038 \approx 4 \times 10^{-3}
\end{aligned}
$$




$$
\begin{aligned}
& +(1-\epsilon)\left[A_{1} B_{2}+\left(1-A_{1} B_{1}\right) G_{1}+\left(1-A_{2} B_{2}\right) g_{2} H_{1} H_{2}\right] \\
& \left.\left[\left(1-\gamma_{2}\right)+\gamma_{2} A_{2} B_{3}+\gamma_{2}\left(1-A_{2} B_{2}\right) G_{2}+\gamma_{2}\left(1-A_{2} B_{2}\right) g_{2} H_{3} H\right]\right]
\end{aligned}
$$

Note that in this equation $(1-a)\left(1-y_{1}\right)$ is not included. This omission is made because the equation is dealing directly wịth failure events due to human error, and therefore it is not necessary to subtract the failure event labeled $Q_{1}$ in Figure 2. Also note that $\left(1-\beta_{2}\right)$ and both $\left(1-\gamma_{2}\right)$ terms are included, for reasons given under the discussion of the indirect approach.

$\mathrm{Q}_{\mathrm{T}_{\mathrm{i}}}$, the system degradation due to human error using the low-range figures from Table III, is calculated as

$$
\begin{aligned}
\mathrm{Q}_{\mathrm{T}_{1}=} & .9[.0003+.9997 \times .002+.9997 \times .998 \times .00005] \\
& {[.2+.8 \times .0003+.8 \times .9997 \times .004+.8 \times .9997 \times .996 \times .0008] } \\
& +.1 \times .9[.9[.0003+.9997 \times .002+.9997 \times .998 \times .00005] \\
& {[.5+.5 \times .0003+.5 \times .9997 \times .004+.5 \times .9997 \times .996 \times .0008] } \\
& +.1[.0003+.9997 \times .1+.9997 \times .9 \times .02] \\
& {[.5+.5 \times .0003+.5 \times .9997 \times .2+.5 \times .9997 \times .8 \times .32]] } \\
= & .9 \times .00048+.09[.9 \times .00118+.1 \times .08611] \\
= & .0013 \approx .10^{-3}
\end{aligned}
$$

' $\mathrm{Q}_{\mathrm{T}_{2}}$, the system degradation due to human error using the high-range figures from Table III, is calculated as

$$
\begin{aligned}
\mathrm{Q}_{\mathrm{T}_{2}}= & .9[.0003+.9997 \times .002+.9997 \times .998 \times .00005] \\
& {[.2+.8 \times .0003+.8 \times .9997 \times .004+.8 \times .9997 \times .996 \times .0008] } \\
& +.1 \times .9[.9[.0003+.9997 \times .02+.9997 \times .98 \times .005] \\
& {[.5+.5 \times .0003+.5 \times .9997 \times .04+.5 \times .9997 \times .96 \times .08] } \\
& \quad .1[.0003+.9997 \times .2+.9997 \times .8 \times .08] \\
& {[.5+.5 \times .0003+.5 \times .9997 \times .4+.5 \times .9997 \times .6 \times .8]] } \\
= & .9 \times .00048+.09[.9 \times .01407+.1 \times .24837] \\
= & .0038 .4 \times 10^{-3}
\end{aligned}
$$


It should not be surprising that the answers for $\mathrm{Q}_{\mathrm{T}_{1}}$ and $\mathrm{Q}_{\mathrm{T}_{2}}$ using the failure probabilities are identical to the answers obtained by using the success probabilities.

\section{An Approximation of the Exact Failure Equation}

Unless one has statistical clerks at his disposal, the above calculations can be tedious. A shortcut approximation can be used and usually will be sufficiently accurate as long as the success probabilities of nearly all events equal at least .99. (The more success probabilities not reaching .99, the less accurate the approximation will be.) Consider the left-hand branch of Figure 2. Failure of this branch, given ground operations, can be approximated as:

$$
\begin{aligned}
Q_{S \mid a} & \left(A_{1} B_{1}+C_{1}+D_{1} D_{2}\right)\left(1-\beta_{2}+A_{2} B_{2}+C_{2}+D_{3} D_{4}\right) \\
& \approx(.0003+.002+.00005)(.2+.0003+.004+.0008) \\
& \approx .00048 \approx 5 \times 1 U^{-4}
\end{aligned}
$$

The answer, .00048 , is identical to the answer obtained with the exact equat1on.

Note that each term in this approximation is the approximate first or second order failure term in the exact failure equation. This approximation is derived by summing the first- and second-order terms which are combinations of failure probab1lities in a. series part of the system where these terms are both sufficient and necessary.* The failure probabilities for the parallel parts of the system must, of course, be multiplied to account for the system redundancy (parallclism). Thus, in the approximate equation above, the first set of terms in parentheses represents a failure on Trial 1 while the second set of terms in parentheses represents a failure on Trial 2. System failure requires a fallure on both Trial 1 and Trial 2 . Thus, thc two sets nf. terms are multiplied to give total system failure rate resulting from human error, given that ground operations occur.

T.f the approxlmale equation is used to calculate the probability of failure resulting from human error of the middle branch of the tree, given $(1-a), \gamma$, , and $\epsilon$, the following equation is relevant:

*In a series combination, only first-order terms would ordinarily be used. For example, in the two-switch series circuit in Table IV, the exact equation for $F$ (failure) is $A B+a B+A b$. But if the probabilities associated with $a$ and $b$ are both about .99, then, as stated above, $a$ and b can be dropped from the equation without introducing a large error in the estimated failure rate. Furthermore, the term $A B$ is sufficient but not necessary. for failure. That is, either $A$ or $B$ alone will result in a failure. Therefore, the approximate failure equation is $\mathrm{F} \approx \mathrm{A}+\mathrm{B}$. In a simple parallel combination, an approximation is not appropriate. For example, in the two-switch parallel circuit shown in Table $I V, F=A B$. Of course, if the probability. of one of these failure events is close to 1.0 , it may'be sufficient to use the lower probability figure as an approximation of ' $F$. 


$$
Q_{S \mid(1-a), \gamma_{1}, \epsilon} \approx\left(A_{2} B_{2}+E_{2}+F_{3} F_{2}\right)\left(1-\gamma_{2}+A_{2} B_{2}+E_{2}+F_{3} F_{4}\right)
$$

and

$$
\begin{aligned}
Q_{\text {Low Range }} & \approx(.0003+.002+.00005)(.5+.0003+.004+.0008) \\
& \approx .0012 \approx .001 \\
\text { Q High Range }_{\text {Hit. }} & (.0003+.02+.005)(.5+.0003+.04+.08) \\
& \approx .0157 \approx .016
\end{aligned}
$$

The unrounded estimates, .0012 and .0157 , can be compared with the corresponding estimates, .0012 and .0141, using the exact failure equations. Thus, the approximation for the low range would be considered adequate even though one of the success probabilities, $\gamma_{2}$, is considerably less than .99 . The approximation is less accurate for the high range since four of the seven terms do not meet the criterion of .99 for their related success probabilities. However, many human-factors specialists would probably consider this approximation to be sufficiently accurate, especially in view of the assumption discussed in Chapter II of doubling error rates after an error has been made.

If the approximate equation is used to calculate the probability of failure resulting from human error of the right-hand branch of the tree, given $(1-a)$; $\gamma_{1}$, and $(1-\epsilon)$, the following equation is relevant:

$$
Q_{S I}(1-a), \gamma_{1},(1-\epsilon) \approx\left(A_{2} B_{2}+G_{1}+H_{1} H_{2}\right)\left(1-\gamma_{2}+A_{2} B_{2}+G_{2}+H_{3} H_{4}\right)
$$

and

$$
\begin{aligned}
Q_{\text {Low Range }} & (.0003+.1+.02)(.5+.0003+.2+.32) \\
& \approx(.1203)(1)^{*} \approx .1203 \approx .1 \\
\text { Q High Range } & \approx(.0003+.2+.08)(.5+.0003+.4+.8) \\
& \approx(.2803)(1)^{*} \approx .2803 \approx .3
\end{aligned}
$$

The unrounded estimates, .1203 and .2803 , can be compared with the corresponding unrounded estimates, .0861 and .2484 , using the exact failure equations, These differences are fairly substantial and some reliability specialists would prefer to

*A probability of error greater than 1.0 is meaningless; therefore 1.0 is used even though the sum of the individual probabilities is greater than 1.0 . As discussed earlier, the value greater than one merely means that the average performance beginning with $\gamma_{2}$ or $1-\gamma_{2}$ under high-stress conditions, after a failure has been experienced following $\gamma_{1}$ under high-stress conditions, will not lead to a successful translation of the code. 
avoid this additional error in the estimate and use the exact equations. Others would note that the differences in error rates, i.e., about .03 for each comparison, are probably not significant in view of the grossness of the estimated error rates for tasks performed under high-stress conditions. In any event, the use of the above type of approximation does yield higher estimates of system degradation, and this fact should be kept in mind.

The following calculation illustrates that when high error rates are associated with events which in themselves have a low probability of occurrence and vice versa, the use of the approximation for estimating total system degradation due to human error does not lead to substantially different results than when using the exact failure equation.

$$
\begin{aligned}
& U_{T} \approx a\left(A_{3} B_{1}+E_{2}+D_{2} D_{2}\right)\left(1-\beta_{2}+A_{2} D_{2}+C_{2}\left(D_{3} D_{4}\right)\right. \\
& +(1-a) \gamma_{1}\left[\epsilon\left(A_{2} B_{1}+E_{1}+F_{1} F_{2}\right)\left(1-\gamma_{2}+A_{2} B_{2}+E_{2}+F_{3} F_{4}\right)\right. \\
& \left.f(1-E)\left(A_{1} B_{2}+G_{1}+H_{1} H_{2}\right)\left(1-y_{2}+A_{2} B_{2}+G_{2}+H_{3} H_{4}\right)\right] \\
& \mathrm{Q}_{\mathrm{T}_{1}} \approx .9(.0003+.002+.00005)(.2+.000 j+.004+.0008) \\
& +.1 \times .9[.9(.0003+.002+.00005)(.5+.0003+.004+.0008) \\
& \left.+.1(.0003+.1+.02)(.5+.0003+.2+.32)^{*}\right] \\
& .0016 \approx 2 \times 10^{-3} \\
& \mathrm{Q}_{\mathrm{T}_{2}} \approx .9(.0003+.002+.00005)(.2+.0003+.004+.0008) \\
& +.1 \times .9[.9(.0003+.02+.005)(.5+.0003+.04+.08) \\
& \left.+.1(.0003+.2+.08)(.5+.0003+.4+.8)^{k}\right] \\
& \approx .0042 \approx 4 \times 10^{-3}
\end{aligned}
$$

\footnotetext{
*See footnote on previous page.
} 
This topic heading provides the final logical step in our application of THERP to a hypothetical operational system. As explained in Chapter I under the same topic heading, the initial procedure in this step is to calculate all $Q_{i}$ values and order them from largest to smallest. $Q_{i}$ is the probability that the system will fail due to some $P_{i}$, the probability that an operation can and is supposed to occur and that it will lead to an error of class $i$. Thus, in this section of the report, the estimated $10^{-3}$ to $4 \times 10^{-3}$ system degradation resulting from human errors will be allocated among the various human error terms in Figure 2 .

\section{Calculation of $Q_{i}$ Values}

We will let $n_{i}$ equal 1.0 , since each $P_{i}$ must be computed separately. Therefore, the equation from Chapter $I, Q_{i}=1-\left(1-F_{i} P_{i}\right)^{n_{i}}$, can be simplified to $Q_{i}=$ $F_{i} P_{i}$ for each $Q_{i}$. In calculating $P_{i}$, however, certain dependencies in the probability tree in Figure 2 must be taken into account. These dependencies stem from the dependence of Trial 2 on the failure of Trial 1 . If the dependencies are not taken'into account, the sum of all the $Q_{i}$ values, $\Sigma Q_{i}$, would not equal $Q_{T}$, the total system degradation resulting from human error.

In order to illustrate how the individual $Q_{i}$ equation can be derived, we will first consider only ground operations, that ia, the lefl-must branch of the probability tree. The best way to avoid errors in deriving the $Q_{i}$ equations is to start out by writing down all of the possible paths to failure, given $a$. The twelve such paths, the sum of which equals $\mathrm{Q}_{\mathrm{T} \mid a}$, are listed below. For simplification each of these twelve is set equal to a single symbol.

$$
\begin{aligned}
& L=\left(A_{1} B_{2}\right)\left(1-\beta_{2}\right) \\
& M=\left(A_{1} B_{2}\right) \beta_{2}\left(A_{2} B_{2}\right) \\
& N=\left(A_{1} B_{1}\right) B_{2}\left(1-A_{2} B_{2}\right) C_{a} \\
& R=\left(A_{1} B_{2}\right) \beta_{2}\left(1-A_{2} B_{2}\right) c_{2} D_{3} D_{4} \\
& S=\left(1-A_{2} B_{2}\right) C_{1}\left(1-\beta_{2}\right) \\
& T=\left(1-A_{1} B_{1}\right) C_{1} \beta_{2} A_{2} B_{2} \\
& U=\left(1-A_{1} B_{1}\right) C_{2} B_{2}\left(1-A_{2} B_{2}\right) C_{2} \\
& V=\left(1-A_{1} B_{1}\right) C_{1} \beta_{i}\left(1-A_{2} B_{2}\right) C_{2} D_{3} D_{4} \\
& W=\left(1-A_{2} B_{2}\right) c_{2} D_{1} D_{2}\left(1-\beta_{2}\right) \\
& X=\left(1-A_{1} B_{1}\right) c_{1} D_{1} D_{2} \beta_{2} A_{2} B_{2} \\
& Y=\left(1-A_{1} B_{1}\right) c_{1} D_{1} D_{2} \beta_{2}\left(1-A_{2} B_{2}\right) C_{2} \\
& Z=\left(1-A_{1} B_{2}\right) c_{2} D_{2} D_{2} \beta_{2}\left(1-A_{2} B_{2}\right) c_{2} D_{3} D_{4}^{*}
\end{aligned}
$$

${ }^{*} \beta_{1}$ and $\zeta$ are dropped from the twelve paths because they both equal 1.0 . 
Consider first the effect of the four error terms $\left(1-\beta_{2}, A_{2} B_{2}, C_{2}\right.$, and $\left.D_{3} D_{4}\right)$ in the second half of the branch, i.e., the Trial 2 terms. ${ }^{*} F_{i}$ for each of these four error terms must equal 1.0 because, in our hypothetical model, there is no chance for system success once any one of them has occurred. All that is left, therefore, is to work out the $P_{i}$ values for each of the four error terms in Trial 2 .

Let us turn to the system failure rate resulting from $D_{3} D_{4}$, the last chance for failure. This $Q_{i}$ will be identified as $Q_{7} \cdot D_{3} D_{4}$ occurs in three paths, $R, V$, and $z$. But one cannot ascribe to $D_{3} D_{4}$ alone the blame for the fallures denoted by these three paths. ${ }^{* *}$ Note that error terms $A_{2} B_{1}, C_{2}$, and $D_{2} D_{2}$ are associated, respectively, with $R, V$, and $Z$. All the other terms in $R, V$, and $Z$ (except, of course, $D_{3} D_{4}$ ) are success terms. Therefore, the system failure rate ascribed to these three paths must be allocated among $D_{3} D_{4}$ and these three Trial 1 error terms. Thus, $D_{3} D_{4}$ gets the blame for

$$
\frac{D_{3} D_{4}}{D_{3} D_{4}+A_{1} B_{1}} \text { of } R \text {, for } \frac{D_{3} D_{4}}{D_{3} D_{4}+C_{1}} \text { of } V \text {, and for } \frac{D_{3} D_{4}}{D_{3} D_{4}+D_{2} D_{2}} \text { of } \mathrm{Z} \text {. }
$$

and the $Q_{i}$ for $D_{3} D_{4}$ can bo written as:

$$
Q_{7}=\frac{D_{3} D_{4} R}{D_{3} D_{4}+A_{1} B_{1}}+\frac{D_{3} D_{4} V}{D_{3} D_{4}+C_{1}}+\frac{D_{3} D_{4} Z}{D_{3} D_{4}+D_{1} D_{2}}
$$

It can be noted, by appropriate factoring, that $R+V+Z$ contains the probability of failure on Trial 1, that is, $A_{1} B_{2}+\left(1-A_{1} B_{1}\right) C_{1}+\left(1-A_{1} B_{1}\right) c_{1} D_{2} D_{2}$. This probability expression reflects the fact that $D_{3} D_{4}$ will not occur unless Trial 1 has failed to produce a successful code translation.

Similarly, the $Q_{i}$ equations for the other Trial 2 error terms are written as:

$Q_{6}$, the $Q_{i}$ for $C_{2}=\frac{C_{2} N}{C_{2}+A_{1} B_{1}}+\frac{C_{2} U}{C_{2}+C_{1}}+\frac{C_{2} Y}{C_{2}+D_{1} D_{2}}$

$Q_{5}$, the $Q_{i}$ for $A_{2} B_{2}=\frac{\dot{A}_{2} B_{2} M}{A_{2} B_{2}+A_{2} B_{2}}+\frac{A_{a} B_{a} T}{A_{2} B_{2} 2 C_{1}}+\frac{A_{2} B_{2} X}{A_{2} B_{2}+D_{1} D_{2}}$

$Q_{4}$, the $Q_{i}$ for $1-\beta_{2}=\frac{\left(1-\beta_{2}\right) L}{\left(1-\beta_{2}\right)+A_{1} B_{1}}+\frac{\left(1-\beta_{2}\right) S}{\left(1-\beta_{2}\right)+C_{1}}+\frac{\left(1-\beta_{2}\right) W}{\left(1-\beta_{2}\right)+D_{1} D_{2}}$

*It will be recalled that $1-\beta_{2}$ is regarded as an error term hecause it can occur only if successful code translation is not attained on Trial 1.

${ }^{* * *}$ If this type of calculation error is made for all the error terms in this branch, then $\Sigma Q_{i}\left|a=2 Q_{T}\right| a$ which is obviously false since $\Sigma Q_{i} \mid a$ must equal $Q_{T} \mid a$. The incorrect answer of $2 \mathrm{Q}_{\mathrm{T}}$ la results from failure to reflect the dependencies in the probability tree between Trial 1 and Trial 2 error terms. Thus, if we denote $F_{i}$ as the probability of failure in Trial 2 , and $F_{j}$ as the probability of failure in Trial 1,

$$
\begin{aligned}
\Sigma Q_{j} \mid a & =A_{2} B_{2} F_{i}+\left(1-A_{2} B_{1}\right) C_{1} F_{i} \\
& +\left(1-A_{1} B_{1}\right) C_{1} D_{1} D_{2} F_{i}+F_{j}\left(1-\beta_{2}\right)+F_{j} \beta_{2} A_{2} B_{2}+F_{j} \beta_{2}\left(1-A_{2} B_{2}\right) C_{2} \\
& +F_{j} \beta_{2}\left(1-A_{2} B_{2}\right) C_{2} D_{3} D_{4} \\
& =F_{i}\left[A_{2} B_{2}+\left(1-A_{1} B_{2}\right)\left(C+C_{2}+C_{1} D_{2} D_{2}\right)\right] \\
& \left.+F_{j} \mid\left(1-\beta_{2}\right)+\beta_{2}\left[A_{2} B_{a}+\left(1-A_{2} B_{2}\right)\left(C_{2}+C_{2} D_{3} D_{4}\right)\right]\right\} \\
& =F_{i} F_{j}+F_{j} F_{i}=2 F_{i} F_{j}=2 Q_{T}
\end{aligned}
$$


It can be noted that each of the above equations contains the Trial 1 failure probability expression.

When we turn to the Trial 1 error terms, it is obvious from the probability tree that any Trial 1 error is not a sufficient cause of system failure. It is still possible for the code translation to be achieved on Trial 2. Thus, $F_{i}$ for each of the three Trial 1 error terms is the probability of failure on Trial 2. Again, in order to avoid errors, it is best to work out the $Q_{i}$ equations by careful reference to the possible failure paths. Consider the $Q_{i}$ for $D_{2} D_{2} . D_{2} D_{2}$ occurs in four paths, $W, X, Y$, and $Z$. But we cannot assign the blame to $D_{2} D_{2}$ alone for these failure paths. The four error terms from Trial 2, i.e., (1- $\left.\beta_{2}\right), A_{2} B, C_{2}$ and $D_{3} D_{4}$, are also associated with, respectively, $W, X, Y$, and $Z$. Therefore, the system failure rate ascribed to these four paths must be allocated among $D_{1} D_{2}$ and the four Trial 2 error items. Thus, $D_{2} D_{2}$ gets the blame for

$\frac{D_{1} D_{2}}{D_{2} D_{2}+\left(1-\beta_{2}\right)}$ of $W$, for $\frac{D_{1} D_{a}}{D_{1} D_{2}+A_{3} B_{2}}$ of $X$, for $\frac{D_{1} D_{2}}{D_{1} D_{2}+C_{2}}$ of $Y$ and for $\frac{D_{1} D_{2}}{D_{2} D_{2}+D_{3} D_{4}}$

of $\mathrm{Z}$; and the $Q_{i}$ and $D_{1} D_{2}$ can be written as:

$$
Q_{3}=\frac{D_{2} D_{2} W}{D_{1} D_{2}+\left(1-\beta_{2}\right)}+\frac{D_{2} D_{2} X}{D_{2} D_{2}+A_{2} B_{2}}+\frac{D_{1} D_{2} Y}{D_{2} D_{2}+C_{2}}+\frac{D_{1} D_{2} Z}{D_{2} D_{2}+D_{3} D_{4}}
$$

It can be noted, by appropriate factoring, that $\mathrm{W}+\mathrm{X}+\mathrm{Y}+\mathrm{Z}$ contains the expression for $F_{i}$, or the probability of failure on Trial 2, i.e., $\left(1-\beta_{2}\right)+\beta_{2} A_{2} B_{2}+$ $\beta_{2}\left(1-A_{2} B_{2}\right)\left(C C_{2}+\beta_{2}\left(1-A_{2} B_{2}\right) C_{2} D_{3} D_{4}\right.$. This same expression is also found in the $Q_{i}$ equations for $C_{2}$ and $A_{2} B_{2}$.

Similarly, the $Q_{i}$ equations for the other Trial 1 error terms are written as:

$$
\begin{aligned}
& Q_{2} \text {, the } Q_{1} \text { for } C_{1},=\frac{C_{2} S}{C_{1}+\left(1-\beta_{2}\right)}+\frac{C_{2} T}{C_{1}+A_{2} B_{2}}+\frac{C_{1} U}{C_{2}+C_{2}}+\frac{C_{2} V}{C_{1}+D_{3} D_{4}} \\
& Q_{2} \text {, the } Q_{i} \text { for } A_{2} B_{2}=\frac{A_{2} B_{2} L}{A_{1} B_{1}+\left(1-\beta_{2}\right)}+\frac{A_{1} B_{1} M}{A_{2} B_{2}+A_{2} B_{2}}+\frac{A_{2} B_{2} N}{A_{1} B_{1}+C_{2}}+\frac{A_{1} B_{2} R}{A_{1} B_{1}+D_{3} D_{4}}
\end{aligned}
$$

The proof is that $\Sigma Q_{i} \mid a=Q_{T} a$.

$$
\begin{aligned}
& \Sigma Q_{1} \mid a=Q_{1}+Q_{2}+\ldots \ldots+Q_{7} \text {; or collecting like terms, } \\
& =\frac{A_{2} B_{2}+D_{3} D_{4}}{A_{2} B_{2}+D_{3} D_{4}} R+\frac{A_{2} B_{2}+C_{2}}{A_{2} B_{2}+C_{2}} N+\frac{A_{2} B_{2}+A_{2} B_{2}}{A_{2} B_{1}+A_{2} B_{2}} M \\
& +\frac{A_{2} B_{2}+\left(1-\beta_{2}\right)}{A_{2} B_{1}+\left(1-\beta_{2}\right)} L+\frac{C_{2}+D_{3} D_{4}}{C_{2}+D_{3} D_{4}} V+\frac{C_{2}+C_{2}}{C_{2}+C_{2}} U \\
& +\frac{C_{1}+A_{2} B_{2}}{C_{2}+A_{2} B_{2}} T+\frac{C_{1}+\left(1-\beta_{2}\right)}{C_{1}+\left(1-\beta_{2}\right)} S+\frac{D_{1} D_{2}+D_{3} D_{4}}{D_{2} D_{2}+D_{3} D_{4}} Z \\
& +\frac{D_{1} D_{2}+C_{2}}{D_{1} D_{2}+C_{2}} Y+\frac{D_{2} D_{2}+A_{2} B_{2}}{D_{2} D_{2}+A_{2} B_{2}} X+\frac{D_{2} D_{2}+\left(1-\beta_{2}\right)}{D_{1} D_{2}+\left(1-\beta_{2}\right.} W \\
& =\mathrm{L}+\mathrm{M}+\mathrm{N}+\mathrm{R}+\mathrm{S}+\mathrm{T}+\mathrm{U}+\mathrm{V}+\mathrm{W}+\mathrm{X}+\mathrm{Y}+\mathrm{Z} \\
& =\mathrm{Q}_{\mathrm{T}} \mid \boldsymbol{a} .
\end{aligned}
$$


There is one final correction to the above set of equations that should be made. Although $1-\beta_{2}$ was regarded as an error term in the calculation of $Q_{T} \mid a$, a $Q_{i}$ value for $I-\beta_{2}$ is not too meaningful if we are concerned primarily with human errors. ${ }^{*}$ It is more reasonable to allocate the $Q_{i}$ calculated for $1-\beta_{2}$ to the $Q_{i}$ values for $A_{3} B_{2}, C_{2}$, and $D_{2} D_{2}$. This allocation reflects the fact that if any of these three Trial 1 error terms occur, the system automatically has a .2 probability of failing since $1-\beta_{2}=.2$. Therefore, the above equations for $Q_{1}$, $Q_{2}$, and $Q_{3}$ are changed, respectively, to drop the fractional modificrs of $L, S$, and $W$. The new $Q_{i}$ equations for $A_{2} B_{1}, C_{1}$, and $D_{2} D_{2}$ now become:

$$
\begin{aligned}
& Q_{2}=L+\frac{A_{1} B_{1} M}{A_{1} B_{1}+A_{2} B_{2}}+\frac{A_{1} B_{1} N}{A_{1} B_{1}+C_{2}}+\frac{A_{1} B_{1} R}{A_{1} B_{1}+D_{3} D_{4}} \\
& Q_{2}=S+\frac{C_{1} T}{C_{1}+A_{2} B_{2}}+\frac{C_{1} U}{C_{1}+C_{2}}+\frac{C_{1} V}{C_{1}+D_{3} D_{4}} \\
& Q_{3}=W+\frac{D_{1} D_{2} X}{D_{2} D_{2}+A_{2} B_{2}}+\frac{D_{1} D_{2} Y}{D_{1} D_{2}+C_{2}}+\frac{D_{2} D_{2} Z}{D_{1} D_{2}+D_{3} D_{1}}
\end{aligned}
$$

The $q_{i}$ equations for the other two branches of the probability tree are similarly derived. Table $V$ presents all the failure paths in the system due to human error. Table VI presents all the $Q_{i}$ equations (in a form suitable for machine calculations) for the reader who wishes to check his own derivations. Table VII presents the $Q_{i}$ values and the data used to determine the values. ** Finally, Table VIII presents selected, rounded $Q_{i}$ values ordered from high to low numbers. Where there is a range for an error rate or for $F_{i}$ or $F_{j}^{\dagger}$, both values are used and are reflected in Tables VI and VII. The $Q_{i}$ for $A_{1} B_{2}$ independent of tree branch is obtained by suming all the $Q_{i}$ values for the $A_{1} B_{1}$ terms in each branch. The $Q_{i}$ for $A_{a} B_{a}$ independent of branch is similarly calculated.

Using the 8-place $Q_{i}$ values in Table VII, it can be shown that the sum of the $Q_{i}$ low-range values (.0013) equals $Q_{T_{1}}(.0013)$ and the sum of the $Q_{i}$ high-range values (.0038) similarly equals $Q_{T_{2}}(.0038)$. Whien the rounded figuroc in $T$ able VIII are used, there is an inconsequential rounding error, but agreement is still obtained at the third decimal place.

\footnotetext{
per se.

*However, one may, if Interested, calculate lhe degradation due to $1-\beta_{2}$

**A1l of the values shown in the tables were obtained by using 8-decimal-place probability figures throughout the calculations and doing the final rounding at the end. This pseudoaccuracy was used merely to check on the closeness of agreement of $\Sigma Q_{i}$ with $Q_{T}$.

$\dagger_{F_{i}}$ is the probability of failure on Trial 2, given a failure on Trial 1 , and $F_{j}$ is the probability of failure on Trial 1 given $a, \epsilon$, or $1-\epsilon$.
} 
TABLE $\mathrm{V}$

The Failure Paths Due to Fuman Error

$$
\begin{aligned}
& \begin{array}{l}
\text { Left Branch } \\
\mathbf{L}=\alpha A_{2} B_{1}\left(1-\beta_{2}\right)
\end{array} \\
& M=a A_{2} B_{1} \beta_{2} A_{2} B_{2} \\
& N=a A_{2} B_{2} \beta_{2}\left(1-A_{2} B_{2}\right) C_{2} \\
& R=\alpha A_{2} B_{2} \beta_{2}\left(1-A_{2} B_{2}\right) c_{2} D_{3} D_{4} \\
& S=a\left(1-A_{2} B_{2}\right) C_{2}\left(1-\beta_{2}\right) \\
& T=a\left(1-A_{1} B_{2}\right) C_{1} \beta_{2} A_{2} B_{2} \\
& U=a\left(1-A_{2} B_{2}\right) C_{2} B_{2}\left(1-A_{2} B_{2}\right) C_{2} \\
& V=a\left(1-A_{1} B_{2}\right) C_{2} B_{2}\left(1-A_{2} B_{2}\right) c_{2} D_{3} D_{4} \\
& W=a\left(1-A_{2} B_{2}\right) c_{2} D_{2} D_{3}\left(1-\beta_{2}\right) \\
& \mathrm{X}=a\left(1-\mathrm{A}_{1} \mathrm{~B}_{2}\right) \mathrm{C}_{2} \mathrm{D}_{2} \mathrm{D}_{2} B_{2} \mathrm{~A}_{2} \mathrm{~B}_{2} \\
& Y=a\left(1-A_{2} B_{2}\right) c_{1} D_{1} D_{2} \beta_{2}\left(1-A_{2} B_{2}\right) C_{2} \\
& Z=a\left(1-A_{2} B_{2}\right) c_{2} D_{2} D_{2} B_{2}\left(1-A_{2} B_{2}\right) C_{2} \cdot D_{3} D_{4}
\end{aligned}
$$

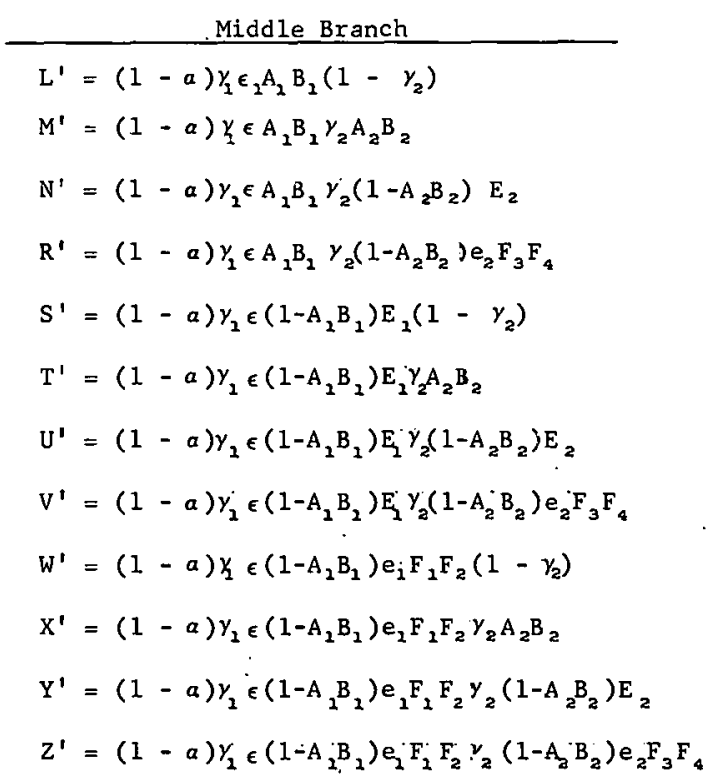

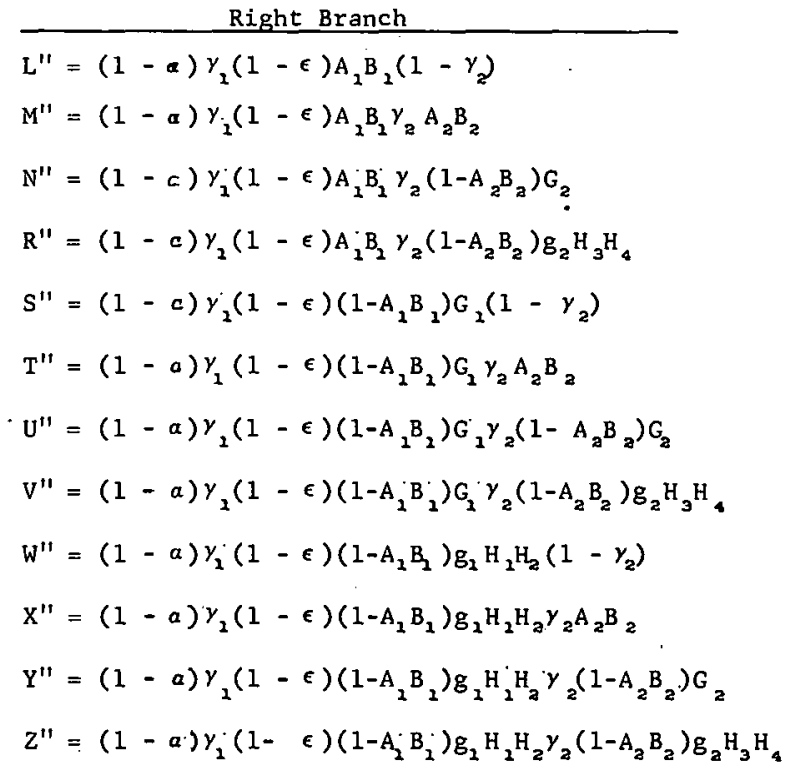


TABLE VI

Equations for $Q_{i}^{*}$

$$
\begin{aligned}
& Q_{1}=L+\frac{A_{2} B_{2} M}{A_{1} B_{1}+A_{2} B_{2}}+\frac{A_{2} B_{1} N}{A_{1} B_{1}+C_{2}}+\frac{A_{1} B_{1} R}{A_{2} B_{2}+D_{3} D_{4}} \\
& Q_{2}=S+\frac{C_{1} T}{C_{2}+A_{2} B_{2}}+\frac{C_{1} U}{C_{2}+C_{2}}+\frac{C_{2} V}{C_{2}+D_{3} D_{4}} \text {. } \\
& Q_{3}=W+\frac{D_{1} D_{2} X}{D_{1} D_{2}+A_{2} B_{2}}+\frac{D_{1} D_{2} Y}{D_{1} D_{2}+C_{2}}+\frac{D_{2} D_{2} Z}{D_{1} D_{2}+D_{3} D_{4}} \\
& * \mathrm{Q}_{4}=\frac{\left(1-\beta_{2}\right) L}{\left(1-\beta_{2}\right)+A_{1} B_{1}}+\frac{\left(1-\beta_{2}\right) S}{\left(1-\beta_{2}\right)+C_{1}}+\frac{\left(1-\beta_{2}\right) W}{\left(1-\beta_{2}\right)+D_{1} D_{2}} \\
& Q_{5}=\frac{A_{2} B_{2} M}{A_{2} B_{2}+A_{1} B_{2}}+\frac{A_{2} B_{2} T}{A_{2} B_{2}+C_{1}}+\frac{A_{2} B_{2} X}{A_{2} B_{2}+D_{1} D_{2}} \\
& Q_{6}=\frac{C_{2} N}{C_{2}+\Lambda_{2} B_{2}}+\frac{C_{2} U}{C_{2}+C_{1}}+\frac{C_{2} Y}{C_{2}+D_{2} D_{2}^{-}} \\
& Q_{7}=\frac{D_{3} D_{4} R}{D_{3} D_{4}+A_{2} B_{1}}+\frac{D_{3} D_{2} V}{D_{3} D_{4}+C_{2}}+\frac{D_{3} D_{4} 7}{D_{3} D_{4}+D_{1} D_{2}} \\
& U_{8}=L^{\prime}+\frac{A_{1} B_{1} M^{\prime}}{A_{1} B_{2}+A_{2} B_{2}}+\frac{A_{1} B_{1} N^{\prime}}{A_{2} B_{1}+E_{2}}+\frac{A_{1} B_{2} R^{\prime}}{A_{2} B_{1} I F_{3} F_{4}} \\
& Q_{9}=S^{\prime}+\frac{E_{2} T^{\prime}}{E_{2}+A_{2} B_{2}}+\frac{E_{1} U^{\prime}}{E_{2}+E_{2}}+\frac{E_{1} V^{\prime}}{E_{1}+F_{3} F_{4}} \\
& Q_{10}=W^{\prime}+\frac{F_{2} F_{2} X^{\prime}}{F_{1} F_{2}+A_{2} B_{2}}+\frac{F_{1} F_{2} Y^{\prime}}{F_{1} F_{2}+E_{2}}+\frac{F_{1} F_{2} Z^{\prime}}{F_{2} F_{2}+F_{3} F_{4}} \\
& { }^{*} \mathrm{Q}_{11}=\frac{\left(1-\gamma_{2}\right) \mathrm{L}^{\prime}}{\left(1-\gamma_{2}\right)+\mathrm{A}_{1} \mathrm{~B}_{1}}+\frac{\left(1-\gamma_{2}\right) \mathrm{S}^{\prime}}{\left(1-\gamma_{2}\right)+\mathrm{E}_{2}}+\frac{\left(1-\gamma_{2}\right) W^{\prime}}{\left(1-\gamma_{2}\right)+\mathrm{F}_{2} \mathrm{~F}_{2}} \\
& Q_{12}=\frac{A_{2} B_{2} M^{\prime}}{A_{2} B_{2}+A_{2} B_{2}}+\frac{A_{2} B_{2} T^{\prime}}{A_{2} B_{2}+E_{2}}+\frac{A_{2} B_{2} X^{\prime}}{A_{2} B_{2}+F_{1} F_{2}} \\
& Q_{13}=\frac{E_{2} N^{\prime}}{E_{2}+A_{1} B_{1}}+\frac{E_{2} U^{\prime}}{E_{2}+E_{1}}+\frac{E_{2} Y^{\prime}}{E_{2}+F_{1} F_{2}} \\
& Q_{14}=\frac{F_{3} F_{4} R^{\prime}}{F_{2} F_{9}+A_{1} B_{1}}+\frac{F_{3} F_{4} V^{\prime}}{F_{3} F_{4}+E_{1}}+\frac{F_{3} F_{4} Z^{\prime}}{F_{3} F_{4}+F_{1} F_{2}} \\
& Q_{15}=L^{\prime \prime}+\frac{A_{1} B_{2} M^{\prime \prime}}{A_{2} B_{2}+A_{2} B_{2}}+\frac{A_{2} B_{2} N^{\prime \prime}}{A_{1} B_{2}+G_{2}}+\frac{A_{1} B_{3} R^{\prime \prime}}{A_{1} B_{1}+H_{3} H_{4}} \\
& Q_{16}=S^{\prime \prime}+\frac{G_{1} T^{\prime \prime}}{G_{1}+A_{2} B_{2}}+\frac{C_{2} U^{\prime \prime}}{G_{1}+G_{2}}+\frac{G_{2} V^{\prime \prime}}{G_{2}+H_{3} H_{4}} \\
& \mathrm{Q}_{17}=\mathrm{W}^{\prime \prime}+\frac{\mathrm{H}_{2} \mathrm{H}_{2} \mathrm{X}^{\prime \prime}}{\mathrm{H}_{1} \mathrm{H}_{2}+\overline{\mathrm{A}}_{2} \overline{\mathrm{B}}_{2}}+\frac{\mathrm{H}_{2} \mathrm{H}_{2} \mathrm{Y}^{\prime \prime}}{\overline{\mathrm{H}_{2}} \overline{\mathrm{H}}_{2}+\mathrm{G}_{2}}+\frac{\mathrm{H}_{2} \mathrm{H}_{2} Z^{\prime \prime}}{\mathrm{H}_{1} \mathrm{H}_{2}+\overline{\mathrm{H}}_{3} \mathrm{H}_{4}} \\
& { }^{*} Q_{18}=\frac{\left(1-\gamma_{2}\right) L^{\prime \prime}}{\left(1-\gamma_{2}\right)+A_{2} B_{1}}+\frac{\left(1-\gamma_{2}\right) S^{\prime \prime}}{\left(1-\gamma_{2}\right)+G_{1}}+\frac{\left(1-\gamma_{2}\right) W^{\prime \prime}}{\left(1-\gamma_{2}\right)+H_{1} H_{2}} \\
& Q_{10}=\frac{A_{2} B_{2} M^{\prime \prime}}{A_{2} B_{2}+A_{2} B_{1}}+\frac{A_{2} B_{2} T^{\prime \prime}}{A_{2} B_{2}+C_{1}}+\frac{A_{2} B_{2} X^{\prime \prime}}{A_{2} B_{2}+H_{1} H_{2}} \\
& Q_{20}=\frac{G_{2} N^{\prime \prime}}{G_{2}+A_{1} B_{1}}+\frac{G_{2} U^{\prime \prime}}{G_{2}+G_{1}}+\frac{G_{2} Y^{\prime \prime}}{G_{2}+H_{1} \tilde{H}_{2}} \\
& \mathrm{Q}_{21}=\frac{\mathrm{H}_{3} \mathrm{H}_{4} \mathrm{R}^{\prime \prime}}{\mathrm{H}_{3} \mathrm{H}_{4}+\mathrm{A}_{1} \mathrm{~B}_{1}}+\frac{\mathrm{H}_{3} \mathrm{H}_{4} \mathrm{~V}^{\prime \prime}}{\mathrm{H}_{3} \mathrm{H}_{4}+\mathrm{G}_{1}}+\frac{\mathrm{H}_{3} \mathrm{H}_{4} \mathrm{Z}^{\prime \prime}}{\mathrm{H}_{3} \mathrm{H}_{4}+\mathrm{H}_{2} \mathrm{H}_{2}}
\end{aligned}
$$

*The first term in the denominator or numerator in the fraction in equation is the error term for that $Q_{i}$.

**These equations are provided for reference purposes. See discussion in text. 
TABLE VII

Hypothetical $Q_{i}$ Values and Rank Order from High to Low

\begin{tabular}{|c|c|c|c|c|c|c|c|c|c|}
\hline \multicolumn{2}{|c|}{$\mathrm{Q}_{\mathrm{i}} \operatorname{Rank}^{*}$} & \multirow[b]{2}{*}{$\mathrm{Q}_{\mathbf{i}}$} & \multirow[b]{2}{*}{ Value $e^{* t}$} & \multirow[b]{2}{*}{ Error Term. } & \multirow{2}{*}{\multicolumn{2}{|c|}{ Error Rate ${ }^{* *}$}} & & \multicolumn{2}{|c|}{ Error Rank } \\
\hline $\mathrm{L}$ & $\overline{\mathrm{H}}$ & & & & & & & $\mathrm{L}$ & $\mathrm{H}$ \\
\hline- & - & $Q_{3}$ & .00005414 & $\mathrm{~A}_{2} \mathrm{~B}_{2} \mid a$ & & $3 \times 10^{-4}$ & & - & - \\
\hline 2 & 3 & $Q_{2}$ & .00036492 & $c_{2}^{2}$ & & $2 \times 10^{-3}$ & & 6 & 9 \\
\hline 6 & 8 & $Q_{3}$ & .00000898 & $\mathrm{D}_{2} \mathrm{D}_{2}$ & & $5 \times 10^{-5}$ & & 9 & 12 \\
\hline- & - & $Q_{4}$ & .00041922 & $\left(1-\beta_{2}\right)$ & & .2 & & - & - \\
\hline- & - & $Q_{5}$ & .00000010 & $\mathrm{~A}_{2} \mathrm{~B}_{2} \mathrm{a}$ & & $3 \times 10^{-4}$ & & - & - \\
\hline 7 & 9 & $Q_{6}$ & .00000478 & $\mathrm{C}_{2}$ & & $4 \times 10^{-3}$ & & 5 & 8 \\
\hline 9 & 10 & $Q_{7}$ & .00000048 & $D_{3} D_{4}$ & & $8 \times 10^{-4}$ & & 7 & 10 \\
\hline- & - & $Q_{8}$ & .00001216 & $A_{1} B_{1} \mid \epsilon$ & & $3 \times 10^{-4}$ & & - & - \\
\hline 4 & 2 & $Q_{9}$ & $\begin{array}{l}.00008115 \\
.00083322\end{array}$ & $E_{1}$ & & $2 \times 10^{-3}, 2$ & $\times 10^{-2}$ & 6 & 6 \\
\hline 8 & 5 & $Q_{10}$ & $\begin{array}{l}.00000202 \\
.00020022\end{array}$ & $\mathrm{~F}_{2} \mathrm{~F}_{2}$ & & $5 \times 10^{-5}, 5$ & $\times 10^{-3}$ & 9 & 7 \\
\hline - & - & $Q_{11}$ & $\begin{array}{l}.00009482 \\
.00098718\end{array}$ & $\left(1-\gamma_{2}\right) \mid \epsilon$ & & & & - & - \\
\hline- & - & $Q_{12}$ & .00000001 & $\mathrm{~A}_{2} \mathrm{~B}_{2} \mid \epsilon$ & & $3 \times 10^{-4}$ & & - & - \\
\hline 10 & 7 & $Q_{13}$ & $\begin{array}{l}.00000027 \\
.00002912\end{array}$ & $\mathrm{E}_{\mathbf{z}}$ & & $4 \times 10^{-3}, 4 \times$ & $10^{-2}$ & 5 & 5 \\
\hline 12 & 6 & $Q_{14}$ & $\begin{array}{l}.00000003 \\
.00006500\end{array}$ & $\mathrm{~F}_{3} \mathrm{~F}_{4}$ & & $8 \times 10^{-4}, 8 \times$ & $10^{-2}$ & 7 & 4 \\
\hline - & - & $Q_{15}$ & .00000135 & $A_{2} B_{2} \mid 1-\epsilon$ & & $3 \times 10^{-4}$ & & - & - \\
\hline 1 & 1 & $\dot{Q}_{16}$ & $\begin{array}{l}.00050739 \\
.00110628\end{array}$ & $G_{1}$ & & $.1, .2$ & & 3 & 3 \\
\hline 4 & 4 & $Q_{17}$ & $\begin{array}{l}.00008369 \\
.00031975\end{array}$ & $\mathrm{H}_{2} \mathrm{H}_{2}$ & - & $2 \times 10^{-2}, 8 \times$ & $10^{-2}$ & 4 & 4 \\
\hline - & - & $Q_{18}$ & $\begin{array}{l}.00045410 \\
.00089221\end{array}$ & $\left(1-\gamma_{2}\right) \mid(1-\epsilon)$ & & & & - & - \\
\hline- & - & $Q_{19}$ & .000000001 & $A_{2} B_{2} \mid(1-\epsilon)$ & & $3 \times 10^{-4}$ & & - & - \\
\hline 5 & 4 & $Q_{20}$ & $\begin{array}{l}.00007495 \\
.00033634\end{array}$ & $\mathrm{G}_{2}$ & & $.2, .4$ & & 2 & 2 \\
\hline 3 & 3 & $Q_{21}$ & $\begin{array}{l}.00010757 \\
.00047164\end{array}$ & $\mathrm{H}_{3} \mathrm{H}_{4}$ & $\cdot$ & $.32, .8$ & & 1 & 1 \\
\hline 5 & 6 & $Q_{22}$ & .00006765 & $A_{2} B_{2}$ & & $3 \times 10^{-4}$ & & 8 & 11 \\
\hline 11 & 11 & $Q_{23}$ & .000000011 & $\mathrm{~A}_{2} \mathrm{~B}_{2}$ & & $3 \times 10^{-4}$ & & 8 & 11 \\
\hline
\end{tabular}

${ }^{*} \mathrm{~L}$ refers to the rank order based on lower estimates of $Q_{i}$ values or error terms, while $\mathrm{H}$ refers to rank order based on the higher estimates. Ranking is based on rounded first significant number. Ranks are not given for $\left(1-\beta_{2}\right)$, and both $\left(1-y_{2}\right)$ terms as these are not human errors and their effects have been included in Trial 1 error terms as discussed in the text. Ranks are given for the complete $A_{1} B_{2}$ and $A_{2} B_{2}$ terms but not for their parts because our interest is in the error terms per se.

* Where two numbers appear, the first is the lower estimate and the second is the higher. The values have been carried to 8-decimal places, merely to aid anyone who wishes to calculate $Q_{i}$ values as an exercise. 


\section{TABLE VIII}

Ranks of High and Low Hypothetical $Q_{i}$ Values

Low Range

\begin{tabular}{rll}
\hline $\begin{array}{c}Q_{i} \\
\text { Rank }\end{array}$ & $\begin{array}{lll}\text { Error } \\
\text { Term }\end{array}$ & $\frac{Q_{i}}{\text { Value }}$ \\
2 & $\mathrm{G}_{1}$ & $5 \times 10^{-4}$ \\
2 & $\mathrm{C}_{1}$ & $4 \times 10^{-4}$ \\
3 & $\mathrm{H}_{3} \mathrm{H}_{4}$ & $10^{-4}$ \\
4 & $\mathrm{E}_{1}$ & $8 \times 10^{-3}$ \\
4 & $\mathrm{H}_{1} \mathrm{H}_{2}$ & $8 \times 10^{-5}$ \\
5 & $\mathrm{G}_{2}$ & $7 \times 10^{-5}$ \\
5 & $\mathrm{~A}_{1} \mathrm{~B}_{1}$ & $7 \times 10^{-5}$ \\
6 & $\mathrm{D}_{1} \mathrm{D}_{2}$ & $9 \times 10^{-6}$ \\
7 & $\mathrm{C}_{2}$ & $5 \times 10^{-6}$ \\
8 & $\mathrm{~F}_{1} \mathrm{~F}_{2}$ & $2 \times 10^{-6}$ \\
9 & $\mathrm{n}_{3} \mathrm{D}_{4}$ & $<10^{-6} * *$ \\
9 & $\mathrm{E}_{2}$ & $<10^{-6}$ \\
9 & $\mathrm{~A}_{2} \mathrm{~B}_{2}$ & $<10^{-6}$ \\
10 & $\mathrm{~F}_{3} \mathrm{~F}_{4}$ & $<<10^{-6 * *}$ \\
$\cdot$ & & $\Sigma Q_{i}=1.3 \times 10^{-3}$
\end{tabular}

High Range

\begin{tabular}{|c|c|c|c|}
\hline $\begin{array}{c}Q_{i} \\
\text { Rank }\end{array}$ & $\begin{array}{l}\text { Error } \\
\text { Term } \\
\end{array}$ & $\begin{array}{c}\mathrm{Q}_{\mathrm{i}} \\
\text { Value }\end{array}$ & $\begin{array}{l}\text { Factor of } \\
\text { Increase }\end{array}$ \\
\hline 1 & $G$ & $10^{-3}$ & 2 \\
\hline 2 & $\mathrm{E}_{1}$ & $8 \times 10^{-4}$ & 10 \\
\hline 3 & $\mathrm{H}_{3} \mathrm{H}_{4}$ & $5 \times 10^{-4}$ & 5 \\
\hline 4 & $C_{1}$ & $4 \times 10^{-4}$ & 0 \\
\hline 5 & $\mathrm{H}_{1} \mathrm{H}_{2}$. & $3 \times 10^{-4}$ & 4 \\
\hline 5 & $G_{2}$ & $3 \times 10^{-4}$ & 4 \\
\hline 6 & $F_{1} F_{2}$ & $2 \times 10^{-4}$ & 100 \\
\hline 7 & $A_{1} B_{1}$ & $7 \times 10^{-5}$ & 0 \\
\hline 7 & $\mathrm{~F}_{3} \mathrm{~F}_{4}$ & $7 \times 10^{-5}$ & .70 \\
\hline 8 & $\mathrm{E}_{3}$ & $3 \times 10^{-5}$ & $>30$ \\
\hline 9 & $D_{1} n_{2}$ & $9 \times 10^{-6}$ & 0 \\
\hline 10 & $\mathrm{C}_{2}$ & $5 \times 10^{-6}$ & 0 \\
\hline 11 & $\mathrm{~A}_{2} \mathrm{~B}_{2}$ & $<10^{-6}$ & 0 \\
\hline 11 & $\mathrm{D}_{3} \mathrm{D}_{4}$ & $<10^{-6}$ & 0 \\
\hline
\end{tabular}

${ }^{*}$ The increase in $Q_{i}$ values as a function of going from low to high range.

** In most human factors applications, one is not justified in expressing any greater accuracy than $<10^{-6}$ or $<<10^{-6}$ to indicate, respectively, $<10^{-6} \geq 10^{-7}$ and $<10^{-?}$ ?

$* * *$ The correct answer is $3.8 \times 10^{-3}$. The difference is due to rounding errors.

Discussion of $Q_{i}$ Values

The ranking of $Q_{i}$ values shows the weakest human 11 nk very clearly. Th1s is the pilot's reception of the code. If one sums the $Q_{i}$ values for the pilot's reception terms $\left(C_{1}, C_{2}, E_{1}, E_{2}, G_{1}, G_{2}\right)$, this conclusion becomes abundantly clear. This sum for the low-range values is approximately $10^{-3}$ and for the high-range values is approximately $2.5 \times 10^{-3}$. These sums constitute roughly from two-thirds to three-quarters ${ }^{*}$ of the total system degradation resulting from human error. But if only the pilot's reception in the air is considered, reception errors ( $E_{1}, E_{2}$, $G_{1}, G_{2}$ ) account for roughly one-half to two-thirds of the total system degradation due to human error. If this amount of degradation were considered unacceptable, then system planners would undoubtedly seek ways either to increase the reliability of the pilot's reception, to bypass him, or change the system in some other way, such as eliminating the requirement to receive the code in the air. The latter

\footnotetext{
${ }^{*}$ In discussing $Q_{i}$ values, it is necessary to keep in mind that our best estimate of certain of the $Q_{i}$ values is a range of values.
} 
change, of course, would pay high dividends inasmuch as it would reduce the system failure rate brought about by $(1-a)\left(1-\gamma_{1}\right)$, i.e., a nonhuman contribution of .01 to system failure. But such gains would have to be balanced against the loss of flexibility introduced by a requirement to receive the code on the ground.

It is interesting to note that the $G$ terms alone constitute roughly one-third of the total system degradation due to human error, and this despite the fact that the right-hand third of the events in the probability tree (which contain the $G$ terms) are estimated to occur with a probability of only $(1-a) \gamma_{1}(1-\epsilon) \approx .009$, or roughly one time in a hundred. It is the experience at Sandia Corporation that uncovering the sometimes unexpected system importance of 10 w probability events is one major benefit from taking the time to set down a complete probability tree and working out the probabilities for each branch. There is an all-too-common tendency to dismiss low probability events, such as the right-hand third of Figure 2 , as being unimportant. The use of the probability tree can bring biases and preconceptions back into line with reality.

In making the recommendations for reducing $\mathrm{Q}_{\mathrm{T}}$ to some acceptable level, one would have to remember that the $\mathrm{Q}_{\mathrm{T}}$ estimated for the hypothetical example is for each system. If one were trying to estimate the total degradation resulting from human errors for all of the systems, he would, of course, have to take into account the estimated number of systems in operation and would have to decide how to assign the probabilities denoted by Greek symbols in Table III to ach of these systems and then how to combine these probabilities appropriately for estimating degradation for all of the systens. For example, some of these probabilities might pertain to one, a few, or many of the systems. Obviously, the single estimated degradation for all systems would be greatly affected by such relationships. 
A technique (THERP) is used to predict human-error rates in a man-machine system and to estimate quantitatively the degradation to the system or any part of it likely to be caused by or associated with the human element. Although the technique is quantitative in nature, its application requires many qualitative judgments by persons who are trained and experienced in the psychology of human beliaviui. However, the close agreement in predictions obtained by two users uf THERP indicates that the judgments that are required may not unduly reduce the consistency of the technique itself. The validity of predictions made with THERP is another question. Very little work has been done in this area, but here, too, there is some evidence that, at least for many applications, validity of the technique and its predictions is adequate. THERP is in its infancy, however, and there is an urgent need for a large data bank of human-error rates taken from operational use and practice exercising of man-machine systems and from the entire manufacturing process related to weapon systems. Along with this need, there is a requirement for continually validating the method and the data upon which it depends.

Several classified applications of THERP have been made to estimate the reliability loss resulting from human errors in selected Air Force aircraft/nuclearweapon systems. These applications have shown a number of human-factors problems and have led to suggestions for revising equipment, procedurcs, and operations which should reduce these problems to an acceptable level. Even though, in thcsc applicatinns, no attempt has been made to claim more accuracy of prediction than a factor of five for tasks performed under normal operational stress (and an order of magnitude accuracy for tasks performed under high-stress conditions), the results of the human-factors reliability analyses have been accepted and recognized by a number of designers and military planners as having value in system planimg.

Finally, the mere attempt to be quantitative forces one to avoid vagueness and to be concrete in thinking about human performance. In applying THERP to analyze man-machine system reliability, Sandia human-factors specialists have often been forced to decide, sometimes to their surprise, that although certain design features deviated considerably from accepted human-engineering practices, their effect upon system reliability would not be important. (Ot course, one should scr1ve fur ds good a human-engineered design as possible, consistent with other system considerations.) Thus, the quantitative approach has forced them to pay more than mere lip service to systems considerations. Perhaps all of us in the human-factors field can benefit from this kind of quantitative approach. 
REF ERENCES

1. Rook, L. W., Jr., "A Method for Evaluating the Human Error Contribution to System Degradation', (unc. paper), in The Proceedings of the Military Operations Research Symposia (MORS), Vo1. 2, No. 1, Part 1, Spring I962, OFF ICIAL USE ONLY.

2. Munger, S. J., Smith, R. W., and Payne, D., An Index of Electronic Equipment Operability: 'Data Store, AIR-C43-1/62-RP(i), American Institute for Research, Pittsburg, Pennsylvania,'January 31,1962

3. Payne, D., and Altman, J. W., An Index of Electronic Equipment Operability: Report of Development, AIR-C-43-1/62-FR, American Institute for Research, Pltisburgh, Penñsylvania, January 31, 1962.

4. Payne, D., Altman, J. W., and Smith, R. W., An Index of Electronic Equipment Operability: Instruction Manual, AIR-C-43-1762-RP(3), American Institute for Research, Pittsburgh, Pennsylvania, January 31 , 1962.

5. Smith, R. W., and Payne, D., An Index of Electronic Equipment Operability: Sample Equipment Evaluation, AIR-C-43-1/62-RP(2), American Institute for Research, Pittsburgh, Pennsylvania, January 31, 1962.

6. Rook, L. W. Jr., Reduction of Human Error in Industrial Production SCTM 93-62(14), Sandia Corporation, Albuquerque, New Mexico, June 1962.

7. Swain, A. D., Human Factors Associated with Prescribed Action Links (U), SCR-634, Sandia Corporation, Albuquerque, New Mexico, May 1963, CONF IDENTIAL DEFENSE INFORMATION.

8. Permissive Device System Reliability Study (U). SWV-3-3003, Air Force Special Weapons Center, Kirtland Air Force Base, New Mexico, no date (distributed in February 1963), SECRET FORMERLY RESTRICTED DATA.

9. "Operational Reliability"; Appendix N, A Preliminary Study of the Bomb Design Problem with Particular References to the TX-12 Weapon (U), VoI. II, SC-2797(TR), Sandia Corporation, Albuquerque, New Mexico, May 1953, SECRET RESTRICTED DATA.

10. Miller, R. B., A Method for Man-Machine Task Analysis, WADC TR 53-137, Wright Air Development Center, Wright-Patterson Air Force Base, Ohio, June 1953.

11. Swain, A. D., System and Task Analysis, A Major Tool for Designing the Personnel Subsystem, SCR-457, Sandia Corporation, Albuquerque, New Mexico, January 1962.

12. Swain, A. D., Altman, J. W., and Rook, L. W., Jr., Human Error Quantification: A Symposium. SCR-610, Sandia Corporation, Albuquerque, New Mexico, Apri1 1963.

13. Meister, D., Individual and System Error in Complex Systems, unpublished memorandum, General Dynamics/Astronautics, San Diego, California, July 10,1962 (presented at the September 1962 convention, American Psychological Association, St. Louis, Missouri).

14. McCornack, R. L., Inspector Accuracy: A Study of the Literature, SCTM 53-61(14), Sandia Corporation, Albuquerque, New Mexico, February 1961.

15. Klier, S., and Linskey, J. W., Selected Abstracts from the Literature on Stress NAVTRADEVCEN 565-1, U. S. Nava1 Training Device Center, Port Washington, New York, November' 1960 . 
16. Grinker, R. R., and Spiege1, J. P., Men Under Stress, McGraw-Hill Book Co., Inc., New York, N. Y., 1963 (reprinted from 1945).

17. Bartlett, F. C., "The Measurement of Human Skil1", Brit. med. J., 1947, 1, 835 and 877 (Reprinted in Occup. Psychol., 1948, 22, 31-38); quoted in Finan, J. L., A Review of Representative Tests Used for the Quantitative Measurements of Behavior-Decrement Under Conditions Related to Aircraft Flight, AFTR-5830, Air Material Command, USAF, Wright-Patterson Air Force Base, Ohio, July 1949, p. 156.

18. Ronan, $W . W$, Training for Emergency Procedures in Multiengine Aircraft, AIR-153-53-FR-44, American Institute for Research, Pittsburgh, Pennsylvania, March 1953. 
DISTRIBUTION:

UC-38 Standard Distribution TID-4500(26th Ed.) (519)

Human Engineering Laboratory

Aberdeen Proving Ground, US Army

Maryland

Human Factors Group, ACF Electronics

ACF Industries, Riverdale, Maryland

Human Factors Staff, ACF Spark Plug Milwaukee, Wisconsin

Human Factors Staff, Reliability Div. Liquid Rocket Plant, Aerojet General Corp. Sacramento, Calif.

Human Engineering Group Aeronautical Research Lab., Dept. of Supply GPO Box 4331, Melbourne, Australia

Library, Space and Weapon Systems Aeronutronic, Ford Road Newport Beach, Calif.

Human Factors Staff, $\Lambda$ trcraft Armaments, Inc. Cockeysville, Md.

Library, American Institute for Research 410 Amberson Ave., Pittsburgh $32, \mathrm{~Pa}$.

Library, American Institute for Research 4020 Buckingham Road, Los Angeles 8, Calif.

Human Factors Staff, Am. Machine \& Foundry 11 Bruce Place, Greenwich, Conn.

Library, Applied Psychology Corp. 41,1,3 Lee Hi.ghway, Arlington 7, Va.

Applied Psychology Research Unit Med. Res. Councll 15 Chaucer Road, Cambridge, England

Library, Applied Psychological Services $114 \mathrm{~N}$. Wayne Blvd., Wayne, $\mathrm{Pa}$.

Applied Science Assocs., Inc. R.D. 3, Box 19A, Valencia, Pa.

Chief, R\&D, Dept. of the Army Washington 25, D.C. Attn: Chief, Human Factors Res. Div.

Human Factors Branch Army Engineer R\&D Lab. Fort Belvoir, Va.

Human Factors References, Armed Services Tech: Info. Agency, Arlington Hall Sta. Arlington $12, \mathrm{Va}$.

Human Factors Project Support, Adv. Systems R\&D Div., Autonetics Div., No. Am. Aviation Anaheim, Calif.

Human Factors Engineering Group Crosley Div., AVCO Corp. 1329 Arlingtón St., Cincinnati, 25, Ohio

Personnel Subsystem Branch, Hq., Ballistic Systems Div., Norton AFB San Bernardino, Calif.

Human Factors Staff, Systems Eng. Div. Battelle Memorial Inst.

505 King Ave.

Columbus 1 , Ohio
Library, Human Engineering Branch Behavioral Sciences Lab. 6570th. Aerospace Med. Res. Labs. Wright-Patterson AFB, Ohio

Human Factors Staff, Aerospace Dept. Aerospace Rockets Div., Bell Aerosystems P.O. Box 1, Buffalo 5, N.Y.

Human Factors, Bell Helicopter Co. P.0. Box 482, Forth Worth 1 , Texas

MH-3224 (Human Factors) BTL, Murray Hil1, N.J.

Library, Engineering Psychology Bioastronautics, Aero. Sp. Div. The Boeing Co., Seattle, Wash.

Library, Personnel Subsystems Mi1. Aircraft Systems Div. The Boeing Co., Seattle, Wash.

Human Factors, Bolt Veranek \& Newman, Inc. 50 Moulton St., Cambridge 38, Mass.

Human Factors Lab., Bostrom Res. Labs. 133 W. Oregon St., Milwaukee 4, Wis.

New Developments Res. Br., Personnel Res. Div., Bureau of Nava1 Personne1 Arlington Navy Annex, Wash. 25, D.C.

Human Engineering, Bureau of Naval Wpns. FWTP-34, Washington 25, D.C.

Human Factors Section, Mil. Sys. Div. Burroughs Corp., Box 305, Paoli, Pa.

Human Factors Section, Burroughs Labs. Box 1901, Colorado Springs, Colo.

Biotechnology Lab., Dept. of Engrg. Univ. of Cal., Los Angeles 24, Calif.

Human Factors Staff, Op. Appli. Lab. A. F. Cambridge Res. Ctr.

L. G. Hanscom Field, Bedford, Mass.

Human Factors Staff, Columbia Univ. Electronics Res. Labs., $632 \mathrm{~W}$. 125th St. New York, N.Y.

Library, Courtney \& Co. Div.

The Matrix Corporation

1630 Pine St., Philadelphia, Pa.

Human Factors Wing, Defense Res. Med. Labs. Box 62, Sta. K, Toronto, Ontario, Canada

Human Factors Staff, Missile \& Space Sys. Div., Saturn Life Sciences Section Douglas Aircraft Co. 3000 Ocean Park Blvd., Santa Monica, Calif.

Human Factors-Manned Spacecraft Missiles \& Space Div., Douglas Aircraft Co. 3000 Osean Park Blvd. Santa Monica, Calif.

Library, Dunlap \& Assocs., In . 429 Ațlantic St., Darien, Conn.

Library, Dunlap \& Assocs., Inc. 1532 Third St., Santa Monica, Calif. 
Human Factors, Ind. Engin. Div.

Eastman Kodak Co., Rochester 4, N.Y.

Human Factors Staff

E. Bollay Assocs.; Inc.

1715 State St., Santa Barbara, Cal.

Library, Entelek Inc.

42 Pleasant St., Newburyport, Mass.

Human Factors Staff, Flight Safety

Foundation, 2871 Sky Harbor Blvd.

Phoenix, Ariz.

Human $\mathrm{F}$ actors Engineering $\mathrm{Br}$. R\&D Group, USA Munitions Command Frankford Arsenal, Phila. 37, Pa

Human Factors Engineering, Life Sciences Sec.. Dept, 594-5, Gen. Dynamics/ Astronautics, P. O Box 1128 San Diego, 12, Calif.

Human Factors Staff Ceneral Dynamics/Convair San Diego, California

Human Factors Lab., Engineering Dept. General Dynamics/Convair Fort Worth, Texas

Human Factors Section, R\&D Dept. Gen. Dynamics/Electric Boat Groton, Conn.

Human Factors Staff Missile \& Sp. Vehicles Dept. GE Co., 3198 Chestnut St. Philadelphia, Pa.

Human Factors Staff, Ordnance Dept. GE Co., Pittsfield, Mass.

Life Sciences Res. Dept. Adv. Terhnology Div. Goodyear Aircraft Corp. 1210 Massillon Rd.., Akron 15, Ohio

Human Fartors Engineering Goodyear Aircraft Corp. Litchfield Park, Arizona

Huilitan Factors 3ection Grumman Aircraft Engrg. Corp. Bethpage, N.Y.

Human F actors, $\mathrm{Sp}$. \& Missile Honeywell Regulator Co. Minneapolis, Minn.

Human Factors Section Operations Dept., HRB-Singer Inc. P.0. Box 60, St. Col1., Pa.

Displays Systems Dept., R\&D Div. Hughes Aircraft Co. Culver City, Calif.

Library, Human Factors Product Effectiveness Labs. Hughes Aircraft, Fullerton, Calif.

Library, Human Factors Res. 1112 Crenshaw Blvd., L.A. 19, Calif.
Library, Human Sciences Res., Inc. 1408 N. Fillmore St., Arlington 1, Va.

Library, Humetrics Div., Thiokol Chem. Corp. 6382 Arizona Circle, L.A. 45, Calif.

Library, HumRRO, AAD Human Res. Unit Ft. Bliss, Texas

Library, USA Aviation Human Res. Unit P.0. Box 428, Ft. Rucker, Alabama

Human Factors Staff, Fed. Sys tems. Div., IBM 10301 Westlake Drive, Rockville, Md.

Human Factors Staff, Mil, Prods. Div. IBM Corp., Owego, N.Y.

Human Factors Staff, IBM Corp. San Jose, Calif.

Human Factors Staff, IBM Research Center Yorktown Heights, N.Y.

Aviation Psychology Lab. U. of Ill. Urbana, Illinois

Human Factors Staff, International Electrial Corp., Box 285, Farantus, N.J.

Human Factors Staff, ITT Fed. Labs. 390 Wash. Ave., Nutley 10, N.J.

Dept. of Psychology, The Johns Hopkins Univ. Baitimore 18 , Md.

Human Factors Staff, Adv. Engrg., Lear Inc. 110 Ionia, Grand Rapids, Mich.

Library, Human Factors, Life Sciences Sec Astronautics Div., Ling-Temco-Vought, Inc. Dallas, Texas

Human Factors Dept., Link Div. General Precision, Inc., Binghamton, N.Y.

Human Factors Staff, Litton Industries 5500 Canoga Ave., Woodland Hills, Calif.

Human Factors Staff (Dept. 72-08) Lockheed-Georgia Co., Marietta, Ga.

Hunan Faclors Staff, Advanced Systems Res. 55-10, Lockheed Míssiles \& Sp. Co. Palo Alto, Calif.

Library, Human Engineering, Space Systems Div. Lockheed Missiles \& Space Co. Sunnyvale, Calif.

Library, Human Factors Dept., Martin Co. Sand Lake Road, Orlando, Florida

Library, Human Factors, Personnel Subsystem Martin-Marietta Co., Box 179, Denver 1, Colo.

Human Factors Dept., Electronic Sys. \& Prods. Div. The Martin Co.,. Mail No. 3092 Baltimore 3 , Md.

Library, The Matrix Corp. 507 18th St., South, Arlington 2, Va.

Engineering Psychology, McDonnell Aircraft Corp., Municipal Airport Box 516 St. Louis 66, Mo. 
Dept : of Psychology,

Univ. of Michigan, Ann Arbor, Mich.

Human Engineering, Space \& Missile Sys Minneapolis-Honeywell Aeronautical Div. 2600 Ridgway Road, Minneapolis, Minn.

MIT Psychology Section Mass. Institute of Technology Cambridge 39 , Mass.

System Sciences Dept., The MITRE Corp. Bedford, Mass.

Systems Research \& Analysis \& Life Sci. Program Manager, Biotechnology \& Human Res., Office of Adv. Res. \& Technology, Hq., NASA, Wash. 5, D.C.

Human Factors, Apol1o Proj. Office Manned Space Craft Center, NASA office City, Houston 1, Texas

Res. Info. Center \& Advisory Service on Info. Processing, Rm. 309, Bldg. 6 Nacional Bureau of steds., Wash. 25 , D.C.

Human Factors Branch, Naval Electronics Lab., San Diego 52, Calif.

Engineering Psy. Br., Naval Res. Lab. Washington 25 , D. C.

Library, Human Factors, Naval Training Device Center, Port Wash., L.I., N.Y.

Industrial Psychology Staff, Dept. of Psychology, U. of N. Mex. Albuquerque, N. Mex.

Human Factors Staff, Norair Div. Northrop Corp., Hawthorne, Calif.

Library, Human Factors, Life Sciences Sec. No. Am. Avaiation, Inc. E. Fifth Ave., Columbus 13, Ohio

Human Factors Analysis, Apollo Life Systems, Space \& Info. Systems Div. Dept. 695-232, No. Am. Aviation, Inc. Downey, Calif.

Behavioral Sciences Labs., Space Labs. Northrop Corp., 1111 E. Broadway Hawthorne, Calif.

Human Factors Unit, Systems Support Dept. Northronics Div., Northrop, Inc. 500 E. Orangethorpe, Anaheim, Calif.

Library, Engineering Psychology Branch Office of Naval Research, Wash. 25, D.C.

Personnel \& Training Res. Br. Office of Naval Research, Wash. 25, D.C.

Library, Psych. Sciences, Office of Scientific Res., U.S. Air Force Washington 25 , D.C.

Laboratory of Aviation Psych. Ohio State Univ., 1314 Kinnear Road Columbus 8 , Ohio

Life Sciences, Operations Res., Inc. 8605 Cameron St., Silver Spring, Md.

Human Factors Staff, Radio Guidance Operations Gen. Electric Co., Syracuse, N.Y.
Human Factors Staff, PHILCO

Govt. \& Industrial Adv. Systems Group 4700 Wissahickon Ave., Phila. 44, Pa.

Human Factors Dept., Philco Western Dv. Labs. 3875 Fabian Way, Palo Alto, Calif.

Human Factors Unit, Engineering Sciences Lab. Picatinny Arsenal, Bldg. 350, Dover, N:J.

Industrial Psychology Staff, Psych. Dept. Purdue Univ., West Lafayette, Indiana

Human Factors Staff, Radio Corp. of Am. B1dg. 10-1-7, Camden 2, N.J.

Library, Human Factors, The RAND Corp. 1700 Main St., Santa Monica, Calif.

Human Factors Staff, Rocketdyne (596-181) Canoga Park, Calif́.

Human Engineering Laboratory Rome Air Dev. Cent. Griffiss AFB Rome, N.Y.

Library, Rowland \& Co. P.O. Box 6l, Haddonfield, N.J.

Human Factors Staff, Reliab. Eng, Elect. Div., Ryan Aeronautical Co., P.o. Box 2308, San Diego 12, Calif.

Serendipity Associates, 14827 Ventura Blvd. Sherman Oaks, Calif.

Research Group in Psych. \& Social Sciences Srithsonian Inst., Washington 25, D.C.

Human Factors Staff, Space Tech. Labs. One Space Park, Redondo Beach, Calif.

Advanced Systems Sec., Dev. Directorate AF SWC, Albuquerque, New Mex.

Systems Analysis Program, Stanford Research Institute, Menlo Park, Calif.

Human Factors Staff, Stromberg-Carlson System, P.O. Box 2449, San Diego 12, Cal.

Dept. of Psych., Univ. of Sidney Sidney, Australia

Human Factors Staff, Sylvania Electronic Systems, 40 Sylvan Rd., Waltham 54, Mass.

Human Engineering Branch, Behavioral Sciences Div., SCGB3, AF Systems Command Andres Air Force Base, Md.

Library, Human Factors, Systems Dev. Corp. 2500 Colorado Ave., Santa Monica, Calif.

Human Factors, Advanced Systems Dept. System Dev. Corp., Lexington 73, Mass.

Library, Hunan Factors, TEMPO, GE Co. Santa Barbara, Calif.

Human Factors Staff, Thompson RamoWooldridge, Inc., Canoga Park, Calif.

Library, Dunlap \& Assoc., Inc I050 3ist St. N.W., Waśhington 4, D.C.

Human Factors Eng. Sec., Army Signal Res.\& Dev. Lab., Fort Monmouth, N.J. 
Institute for Psy. Res.

Tufts Univ., Medford 55, Mass.

Human Factors Staff, Weather Systems Center United Aircraft Corp., 800 Main St. East Hartford 8, Conn.

Personnel Subsystems $\mathrm{Br}$. United Technology Center United Aircraft Corp. Sunnyvale, Calif.

Human Factors, Westinghouse Elec. Corp. P.0. Box 746, Baltimore 2, Md.

Dr. James W. Altman Am. Inst. for Res., 410 Amberson Ave. Pittsburgh 32, $\mathrm{Pa}$.

Dr. Nancy S. Anderson Dept. of Psych., Univ. of Md. College Park, Md.

Dr. Richard C. Atkinson Inst, for Math. Studies in the Soc. Scicnces, Stanford Univ. Palo Alto, Calif.

Mr. Charles A. Baker, M111s Sta. 604 Space \& Armament System, Minneapolis-Honeywell Aeronautical Div. 2600. Ridgeway Rd., Minneapolis 40, Minn.

Mr. Harold A. Bauer, Human Factors Dept. Electronics Systems \& Products Div. Mail No. G-3092, Martin Co. Baltimore 3, Md.

Mr. Charles Bates, Jr. Human Engrg. Branch Behavioral Sciences Lab. ASBSER, Wright-Patterson AFB, Ohio

Dr. William C. Biel Systems Dev. Corp., 2500 Colo. Ave. Santa Monica, Calif.

Dr. Robert E. Blanchard Duilán \& Assui. Ini. 1532 Third St., Santa Monica, Calif.

Dr. R. Darrell Bock, Psychometric Lab. Univ. of No. Car., Chapel Hill, N.C.

Prot. Nickolas A. Bond, Ir. Sacramento State College 6000 Jay St., Sacramento, Calif.

Dr. Charles W. Bray Research Group in Psych. \& the Social Sciences, Smithsonian Inst. Washington 25 , D.C.

Dr. Alan A. Burrows, Life Sciences Aircraft Div., Douglas Aircraft Co. Lakewood Blvd., Long Beach, Calif.

Dr. Armand $N$. Chambers io50 3 Ist St. N.W. Washington, D. C.

Prof. Alphonse Chapanis, Dept. of Psych. The Johns Hopkins Univ. Baltimore 18 , Md.
Dr. Julien M. Christensen

Human Engineering $\mathrm{Br}$., Behavioral Sciences Lab., Wright-Patterson AFB, Ohio

Mr. John F. Christian, Human Factors Res. Support Systems Div., Res. Directorate office of Civil Defense, Pentagon Washington 25, D.C.

Lt. Col. Luther C. Cox AFW Lab., KAFB, New Mexico

Col. Anthony Debons Directorate of System Design Electronic System Div., USAF Hanscom Field, Mass.

Dr. Stanley Deutsch, Systems Res. \& Analysis $\&$ Life Sciences, Biotechnology \& Human Res., Hq., NASA, Washington 5, D.C.

Dr. Jack W. Dunlap, Dunlap \& Assoc, Inc. 429 Atlantic Street, Darien, Conn.

Mr. Rohert. T. Fckenrode Dunlap \& Assoc. Inc. 429 Atlantir. St., Dariun, Com.

Wr. Gordon A. Éckstrand, 'líaining Pśych B̈r., Behavioral Sciences Lab. Wright Patterson AFB, Ohio

Mr. Milton A. Fabré, Bldg. 60, Rm. 2937 Space Tech. Labs., One Space Park Redondo Beach, Calif.

Mr. Richard A. Faust, Jr. Rowland and Co!, P. O. Box 61 Haddonfield, N.J.

Dr. Charles A. Fenwick, Dev. Div. C $5200 \mathrm{C}$ Ave. N.E., Cedar Rapids, Iowa

Dr. Sidney I. Firstman, The RAND Co. 1700 Main SL., Sanla Munica, Calir.

Dr. Paul M. Fitts, Dept. of Psych. Univ. of Mich., Ann Arbor, Mich.

Dr. Robert Fitzpatrick Man-Machine Design Group, Norad Dcpt. SDC, 45 Hartwell Ave., Lexington 73, Mass.

Dr. Jnhn C. Flanagan, Am. Tnst. for Res. 410 Amberson Àve., "Pittsburgh 32, $\mathrm{Pa}$.

Dr. John D. Folley, Jr., Applied Science Assoc, Inc. R.D. No. 3, Box 19A, Valencia, Pa.

Mr. R. O. Frantik, 3/126A, Aerospace Corp. P.O. Box 95085, Los Angeles 45, Calif.

Dr. Alvyn M. Freed, Human Factors Staff Reliab. Div, Liquid Rocket Plant Aerojet-Gen. Corp., Sacramento, Calif.

Dr. Melvin Freitag, Genera1 Dynamics/Astronautics San Diego 12, California

Mr. Wesley L. Gaddis, Aerojet-Gen'1. Corp. Reliab. Dept., 1100 W. Hollyvale Ave. Azusa, Calif. 
Dr. Walter F, Grether, Behavioral Sci. Lab. Aerospace Med. Res. Labs.

Wright-Patterson AFB, Ohio

Dr. Nehemiah Jordon, The RAND Corp. 1700 Main St., Santa Monica, Calif.

Mr. Robert Kinch, Western Elec. Co. Winston-Salem, No. Carolina

Dr. Edmund T. Klemmer, MH-3224 Be11 Te1. Labs., Murray Hi11, N.J.

Dr. Martin I. Kurke, c/o Tech. Operations, Inc P.0. Box 116, Fort Belvoir, Va.

Mr. Harald R. Leuba, ARINC Res. Corp. 1700 K St. N.W., Wash. 6, D.C.

Mr. A. D. Levandowsky, Human Factors \& Opers. Analysis Lab., Philco Corp. 3875 Fabian Way, Palo Alto, Calif.

Mr. Stanley Lippert, Adv. I'ech. Dept. Aircraft Div., Douglas Aircraft, Inc . 3885 Lakewood Blvd., Long Beach, Calif.

Dr. Max W. Lund, Human Factors Studies Office of Manned Spaceflight, NASA Washington 25 , D.C.

Prof. John Lyman, Biotechnology Lab. UCLA, Los Angeles 24, Calif.

Dr. Robert R. Mackie, Human Factors Res. 1112 Crenshaw B1vd., Los Angeles 19, Calif.

Capt. Melvin S. Majesty, BSOSP/Capt. Majesty/ 7081. Hq. Bailistic Systems Div, , AFSC USAF, Norton AFB, Calif.

Dr. William McClelland, Human Research office, George Wash. Univ. $300 \mathrm{~N}$. Wash., Alexandria, Va.

Dr. Ernest J. McCormick, Psych. Dept. Purdue Univ., W. Lafayette, Indiana

Dr. David Meister, Dept. 652-4 Gen. Dynamics/Astronautics P.O. Box 1128, San Diego 12, Calif.

Dr. Arthur W. Melton, Dept, of Psych. Univ. of Mich., Ann Arbor, Mich.

Dr. Robert B. Miller, Product Dev. Lab . IBM Corp., Poughkeepsie, N.Y.

Mr. Herber G. Moore, Human Factors off. off. of systems Studies, Off. of Manned Space Flight, NASA Washington 25, D.C.

Dr. Jesse Orlansky, Res. \& Engrg. Support Div. Institute for Defense Analysis, 1666 Conn. Ave., NW Washington 9, D.C.

Col. Howard L. Parris 13907 Woodthorpe Lane Houston, Texas

Dr. Daniel Howland, Dept. of Ind. Engrg. Ohio State Univ.

Columbus 10 , Ohio
Lt. Col. John V. Patterson Personnel Subsystem Div., Minuteman Sys. Program Off., USAF, Ballistic Sys. Div. Norton AFB, Calif.

Mr. George J. Pearson

E. Bollay Assoc., Inc.

1715 State St., Santa Barbara, Calif.

Dr. Evan W. Pickre1, A3-860 MSSD

Douglas Aircraft Co.

Huntington Beach, Calif.

Dr. Gerald F. Rabideau, Human Factors Analysis, Apollo Life Systems, Space \&

Inf1. Systems Div., Dept. 695-232 No. Am. Aviation, Inc., Downey, Calif.

Dr. Maurice Rappaport Agnews State Hospital San Jose, Calif.

Prof. Joseph W. Rigney, Dept. of Psych. Univ. of So. Calif., 3518 Univ. Ave. Los Angeles 7, Calif.

Co1. James II. Ritter Off. of Res. Analyses, Hq. Off. of Aerospace Res., Holloman AFB, N. Mex.

Mr. Paul G. Ronco, Human Engrg. Info. \& Analyses Service, Institute for Psychol. Res., Tufts Univ., Medford 55, Mass.

Dr. Stanley N. Roscoe, Display Sys. Dept. R\&D Div., Aerospace Group Hughes Aircraft Co., Culver City, Calif.

Mr. Ernest Sadler, Dept. 3042-22 Autonetics, P. n. Rnx R-3, Anaheim, Calif.

Dr. Ezra V. Saul, Inst. for Psychol. Res. Tufts Univ., Medford 55, Mass.

Mr. Joseph L. Seminara, Human Factors Staff Adv. Systems Res. 55-10, Lockheed Missiles $\&$ Space Co., Palo Alto, Calif.

Mr. Albert Shapero, Systems Analysis Program Stanford Res. Institute, Menlo Park, Calif.

Dr. Charles W. Simon, Human Factors Society 1532 Third St., Santa Monica, Calif.

Dr. H. Wallace Sinaiko, Res. \& Engrg. Support Div., Institute for Defense Analysis 1666 Conn. Ave. NW, Wash. 9, D.C.

Mr. Edward B. Slebodnick, Boeing Pacific Test Center, The Boeing Co., P.O. Box 1626 Vandenberg $A F B$, Calif.

Mr. J. Albert Southern, Bioastronautics Br. Aeronautical Systems Div. Wright-Patterson AFB, Ohio

Mr. David Stea, Psycho1. Dept Stanford Univ., Stanford, Calif.

Dr. John K. Sterrett, Code NESA, Box 70 NORAD, Ent AFB, Colorado Springs, Colo.

Dr. Warren H. Teichner, Inst. of Environmental Psychophysiology, Univ. of Mass. Anherst, Mass. 
Dr. Martin A. Tolcott, Dunlap \& Assoc, Inc. 429 Atlantic St., Darien, Conn.

Dr. Theodore R. Vallance, SORO, Am. Univ. 5010 Wisconsin Ave., NW, Wash. 16, D.C.

Dr. Harold P. Van Cott, System 473L Command Control Dept. Fed. Systems Div., IBM Corp. 7900 Wisconsin Ave., Bethesda 14, Md.

Dr. Robert Y. Walker, Pilotless Aircraft Div. The Boeing Co., Seattle, Wash.

Dr. Melvin J. Warrick, Human Engrg. Br. Behavioral Sci. Lab. Wright-Patterson AFB, Ohio

Cdr. D. H. Weidig, USS Bon Homme Richard (CVA-31), C/ 1 FPO, San Francisco, Calif.

Prof. Delos D. Wickens, Dept. of Psych. Ohio State Univ., Columbus 10, Ohio

Mr. J. S. Wilinsom, Western Electric Co. Merrimack Valley Works

1600 Osgood St., North Andover, Mass.

Mr. Joseph G. Wohl, Dunlap \& $\triangle$ ssoc., Inc. 429 Atlantic St., Darien, Conn.

Mr. Kenneth E. Wright, Personnel Subsystems Branch, United Technology Ctr.

United Aircraft Corp., Sunnyvale, Calif.

Dr. Joseph W. Wulfeck, Dunlap \& Assoc., Inc. 1532 Third St., Santa Monica, Calif.

Mr. Charles E. Zeleny (Applied Psych. Corp.) 3917 E. Broadway, Tucson, Ariz.

Mr. Henry P. Organ, Personnel Subsystem Lockheed Missiles \& Space Co. Sunnyvale, Calif.

Mr. John Kolb, Associate Editor Product Engrg.

330 West 42 nd St., New York 36, N.Y.

Mr. Joe D. Brower, Human Factors-Manned Spucecrult, Duuglas Aircraft Co. 3000 Ocean'Park Blvd., Santa Monica, Calif.

Mr. Stephen D. Benson, 433L Personnel Subsys." United Aircratt Corp., Weather Sys. Cienter East Hartford 8 , Conn.

Miss Kay K. Anderson, Industrial Engrg. Div. Eastman Kodak Co., Rochester 4, N.Y.

Mr. Fred A. Brooks, Jr., Dunlap \& Assocs. Inc., 42513 th St., NW, Wash. 4, D.C.

Mr. Joel Cooper 444 Calle Mayor, Redondo Beach, Calif.

Dr. Roy D. Dunlap, Tech. Staff Space Technology Labs. One Space Park, Redondo Beach, Calif.

Mr. Joseph T. Fucigna, Jr. Dunlap \& Assoc., Inc.

429 Atlantic St., Stamford, Conn.
Dr. John E. Mangelsdorf, Human Engineering Space Systems Div., Lockheed Missiles and Space Co., Sunnyvale, Calif:

Dr. George L. Murphy, Humetrics Div. Thiokol Chem. Corp. 6382 Arizona Cir., Los Angeles 45, Calif.

Mr. George A. Peters Human Factors Staff, Rocketdyne (596-181) Canoga Park, Calif.

Di. Geusge E. Kuwland, Ruwland and Cu. P.O. Box 61, Haddonfield, N.J.

Mr. Lionel Slater 79 Perry St., New York 14, N.Y.

Mr. H. M. Bryant, US Nava1 Ord. Lab. Corona, Calif.

Mr. L. R. Buchanan 1043 East Spruce, Detroit, Mich.

Mr. C. W. Burgener, Unidynamics Marion, Ill.

Mr. De1igeorges, USN Underwater Sound Lab. Fort Trumbul1, New London, Conn.

Lt. Col. R. L. Forkner, USAF FC/DASA Chief, QA Liaison Detachment Sandia Base, Albuquerque, N. Mex.

Mr. L. G. Fredrick General Electric, Syracuse, N.Y.

Mr. Wilmot M. Gibson Rock Island Arsenal, Rock Island, Ill.

Mr. Dona1d L. Glass, Atomics International P.O. Box 309, Canoga Park, Calif.

Mr. Cerald Conda, Gurr-Rrown Res. Corp. Tucson, Arizona

Mr. W. B. Huber, Adcole Corp. 186 Mass. Ave., Cambridge 39, Mass.

M: David R. Jülins, Gúdyear Aircrall Curp Litchfield Park, Ariz.

Maj.A. J. Klingon, USAF, QA Liaison Det. FC/DASA, Sandía Base, Álbuq., N.Mex.

Mr. N. Lawrence, Link Division Gen. Precision, Inc., Binghamton; N.Y.

Mr. Eugene A. Levin, Ampex Corp. Audio Div., 1020 Kifer Road Sunnyvale, Calif.

Mr. Willie C. Livingston, Goodyear Air. Corp. Litchfield Park, Ariz.

Mr. S. E. Maynard, AiResearch Mfg. Co. Phoenix, Arizona

Mr. J. F. McDonne11, Jr. General Dynamics Corp., Pomona, Calif.

Mr. E. C. Mooney, Hi-Q Div. Aerovoz Corp., Olean, N.Y. 
Mr. Marvin L. Murphy, Texas Instruments, Inc. 3609 Buffalo Speedway, Houston, Texas

Mr. Jack Nelson, The Boeing Company Wichita, Kansas

Mr. Robert Owen

Mitre Corp., Bedford, Mass.

Mr. Robert Poffenberger, Ward Leonard Elec. Co., Hagerstown, Md.

Mr. Jules Rosenbaum 12316-1/2 Pacific Ave., Los Angeles 66, Cal.

Mr. R. M. Salestrom Dale Electronics, Columbus, Neb.

Mr. C. O. Schaefer Schlumberger Well Survey Corp. 5000 Gulf Freeway, Houston, Texas

Mr. Henry E. Vaiden, Bell Tel. Labs. Winston-Salem, No. Carolina

Mr. W. Weckel, Bendix System Div. Ann Arbor, Mich.

Mr. Robert H. White Jet Propulsion Lab., Pasadena, Calif.

Atomic Energy Commission Oak Ridge, Tennessee

Dr. Robert E. Ehrlich, TEMPO General Electric Co. 735 State St. Santa Barbara, Calif.

Dr. A. J. Latham, Acronautico and Missilcs Div. Chance Vought Corp.

P. 0. Box 5907, Dallas 22, Texas

Mr. Harold R. Willis, Mail \# A-182

Martin Company

Denver, Colorado

Capt. Wm. F. Madden, USN

BuMed 513, Navy Dept.

Washington $25, \mathrm{D}$. C.

Mr. C. A. Northend Lockheed Missiles \& Space Co.

Dept. 65-82 Bldg. 523

Sunnyvale, Calif.

Dr. Carl E. Helm, Dept. Psychology Princeton University

Princeton, New Jersey

Mr. H. A. Koch, Human Factors

United Technology Center

Sunnyvale, Calif.

Mr: J. L. Hochman, 8459/539

Lockheed Missiles \& Space Co. Sunnyvale, Calif.

Mr. Kenneth W. Yarnold Dunlap and Associates, Inc. Darien, Conn.

Mr. D. B. Parker, A3-860 MSSD Douglas Aircraft Co. Huntington Beach, Calif.
Dr. James F. Dixon III

Electronics System Labor̂atory, M.I.T. Cambridge 39 , Mass.

Mr. Mayer S. Fistal

Electronics Department

Hamilton Standard

Broadbrook, Conn.

Sandia Corporation Distribution:

E. H. Draper, 1000

L. A. Hopkins, 1300

C. B. McCampbell, 1310

J. P. Cody, 1320

J. H. Findlay, 1400

G. W. Rodgers, 1420

P. J. Komen, 1422

A. B. Church, 1433-1

J. W. Wiesen, 1440

C. S. Williams, 1442

F. W. Muller, 1442-1

R. E. Butler, 1443

A. D. Swain, 1443-2 (50 copies)

H. E. Walker, 1443-2

L. D. Smith, 1500

J. W. Jones, 1510

R. S. Wilson, 1520

H. H. Patterson, 1530

W. L. Stevens, 1531

D. M. O1son, 1532

C. S. Selvage, 1533

S. A. Moore, 1540

I. M. Moore, 2310

G. C. Shelton, 2311-2

R, A. Knapp, 2330

L. J. Paddison, 2400

B. O. Allen, 2442

T. F. Meigs, 2442-1

O. L. Oren, 1551

D. D. Sheldon, 2561

D. G. Letbetter, 4541-3

L. E. Hollingsworth, 7200

D. B. Shuster, 7400

A. F. Cone, 2110

L. Gutierrez, 8100

C. A. Scott, 8118-2

G. W. Anderson, 8140

o. H. Schreiber, 8142-3

D. R. Cotter, 9100

L. W. Rook, Jr., 9100 Staff

A. A. Lieber, 9120

C. Winter, 9130

B. R. Allen, 3421

M. G. Randle, $3428-1$, B1dg. 880

J. M. Zanetti, 3412

L. D. Patterson, 3411-1 (37) 


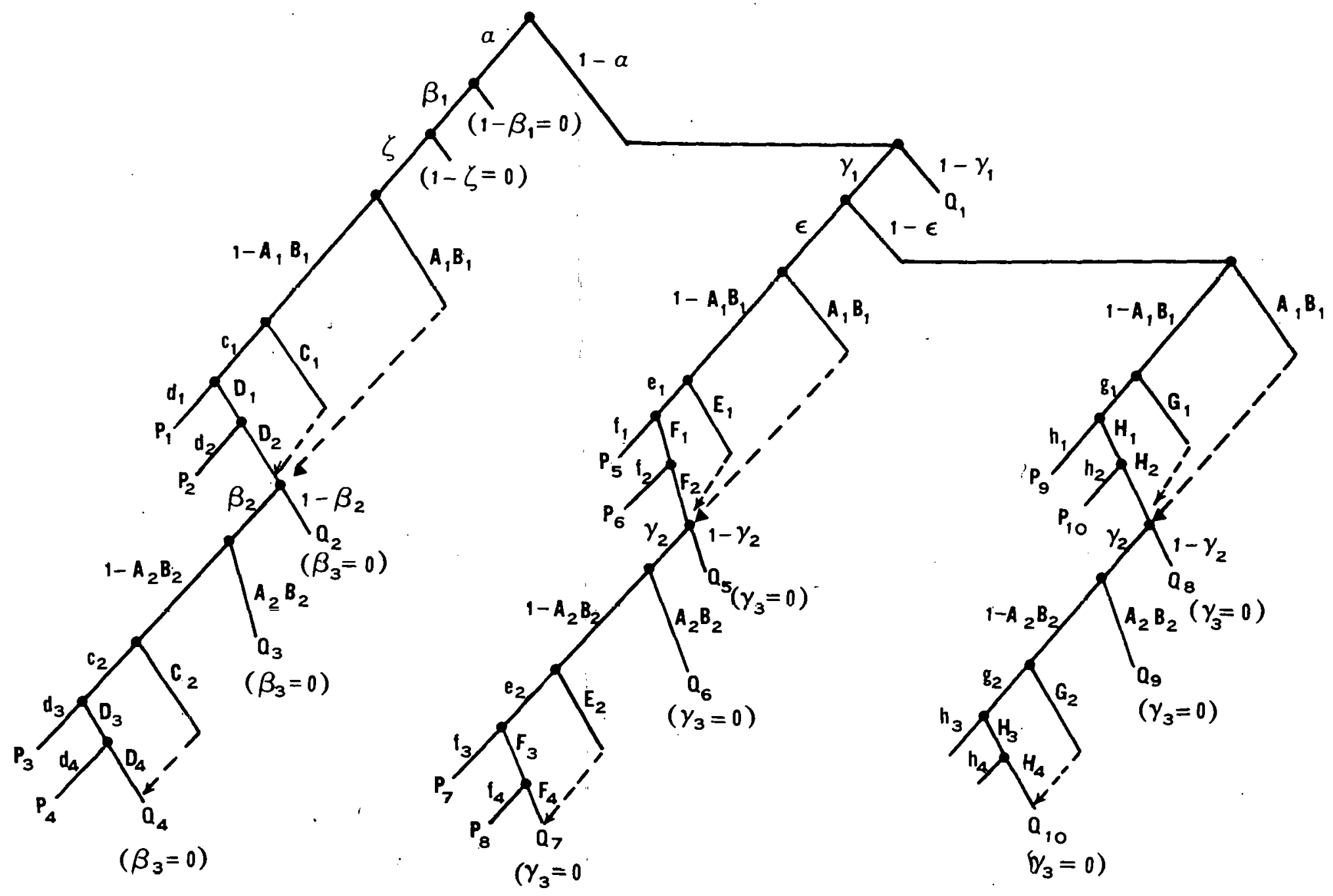

